\title{
Die Entwicklung der Ohrkapsel und des schalleitenden Apparates von Hyla arborea japonica.
}

\author{
Von \\ Sanichi Miyawaki. \\ Aus dem Anatomischen Institut der Keio Universität, Tokyo
}

Mit 25 Textfiguren.

\section{Einleitung.}

Die Entwicklung der Ohrkapsel von Anuren hatten viele Forscher, wie Goette ('75), Stöhr ('79), Gaupp ('93), Peter ('10), Seter ('21-'22) u:a.m. bereits eingehend untersucht und wach ihnen ist das Vorhandensein der zwei Foramina acustica an der medialen Ohrkapselwand als die Norm beobachtet worden. Aber neuerdings fand ich ('27) bei Hyla arborea japonica deren drei. Damit erkennt man einen neuen Anurentyp, wo der Ramus medius des Acusticus, der durch das Foramen acusticum medium passiert, vom Ramus posterior sich verzweigt und immer zur Lagena geht. In der vorliegenden Untersuchung habe ich die Ohrkapsel dieses Tieres entwicklungsgeschichtlich verfolgt und zugleich auch die Entstehung des schalleitenden Apparates.

\section{Material und Methode.}

Ich untersuchte viele larvale Stadien der Hyla arborea japonica Günther und das ausgewachsene Exemplar. Sie wurden mit 10\% Formalin oder Formolalkohol fixiert. Zur Entkalkung des ausgewachsenen Tieres wurden $3 \%$ Salpetersäure angewendet. Nachdem sie in Zelloidin und in Paraffin eingebettet waren, wurden sie alle in frontale Schnittserien von 20-25 $\mu$ Dicke zerlegt und mit Hämatoxylin-Eosin und Eisen- 
hämatoxylin nach Weigert gefärbt. Ich habe 7 Wachsplattenmodelle 33-40 mal vergrössert angefertigt. Für die freundliche Überlassung der schwierig zu beschaffenden Materialien möchte ich hier Herrn Prof. Y. Okada meinen verbindlichsten Dank aussprechen.

\section{Übersicht der rekonstruierten Modelle.}

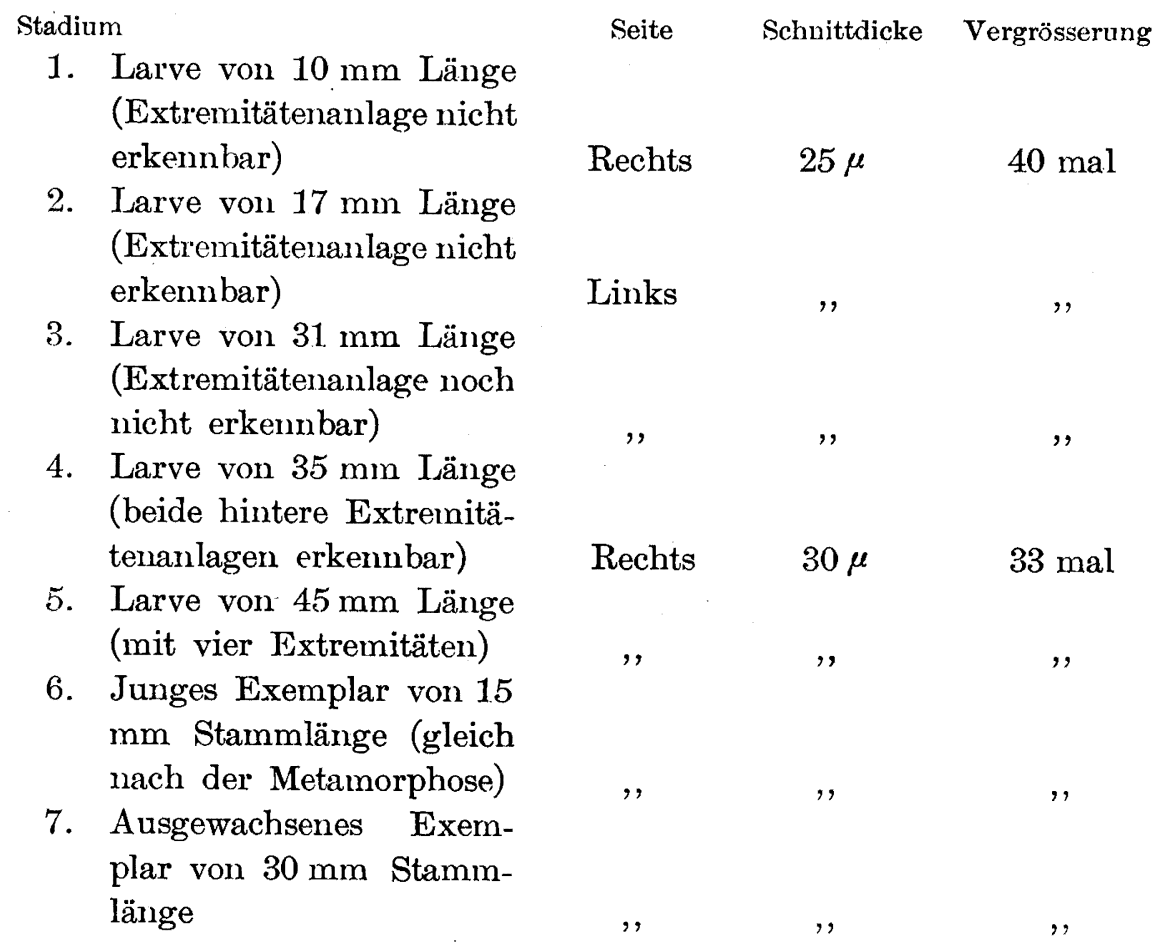

\section{Stadienbeschreibung.}

1. Stadium 1.

Ganze Körperlänge $10 \mathrm{~mm}$, Stammlänge $4 \mathrm{~mm}$, Schwanzlänge $6 \mathrm{~mm}$. Die Augenbläschen sind gut bemerkbar.

Aus dem rechten Ohr wurde ein 40 mal vergrössertes Wachsplattenmodell angefertigt; sein anteroposteriorer Durchmesser beträgt $35 \mathrm{~mm}$ $(0,81 \mathrm{~mm})$, der mediolaterale $20 \mathrm{~mm}(0,5 \mathrm{~mm})$ und der dorsoventrale $13 \mathrm{~mm}(0,81 \mathrm{~mm})$.

Die dorsale Wand der Ohrkapsel hat eine fast rundliche Form und ist stark nach aussen geneigt. Ihr mittlerer Abschnitt ist stark nach innen unten konkav und geht von hier allmählich in den äusseren und 
inneren abgerundeten Rand des Daches über. Das Tectum synoticum ist schon ausgebildet und liegt auf der dem Sinus superior entsprechenden Strecke der Kapsel beschränkt. Der laterale Bogengang ist ziemlich stark nach aussen abgerundet. Durch den Sinus superior wird die mediale Kante der dorsalen Wand in zwei Teile geteilt: einen vorderen kleineren, der nach vorn aussen verlaufend in die vordere Kuppel übergeht, dem vorderen Bogengang entsprechend, und einen grösseren, nach hinten aussen gerichteten, der sich in die hintere Kuppel fortsetzt und in sich

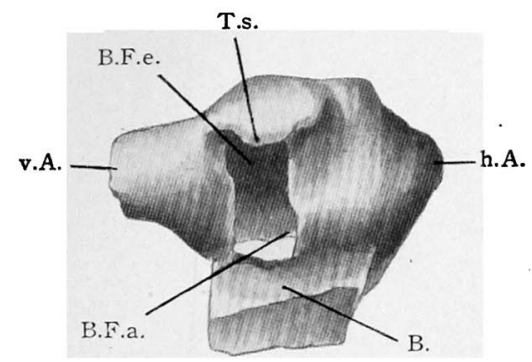

Fig. 1.

Mediale Ansicht des Modells der rechten Ohrkapsel. Stadium 1, $40 \mathrm{mal}$ vergrössert modelliert. den'hinteren Bogengang beherbergt.

Die mediale Wand der periotischen Kapsel wird noch nicht vollständig ausgebildet. An ihrem mittleren Abschnitt befindet sich eine grosse länglich-viereckige Lücke, die fast vertikal von oben nach unten steht. Sie entspricht der Stelle der Foramina acustica und Foramen endolymphaticum in späteren Stadien und ihre grösste Länge beträgt $14 \mathrm{~mm}(0,35 \mathrm{~mm})$, ihre Breite $6 \mathrm{~mm}(0,15 \mathrm{~mm})$ am Boden. Der

hintere Abschnitt dieser Wand verbindet sich mit der Basalplatte, während der vordere diese noch nicht erreicht.

Die ventrale Wand ist medial mit der Basalplatte verbunden und endet aussen mit einem scharfen, freien Rande, die untere Begrenzung des Foramen ovale bildend. Dieses hat an lateraler Wand die Form einer länglichen Spalte, die sich von vorn nach hinten in der Nähe des Bodens, Seine obere Begrenzung ist durch die horizontale Fläche, den unteren Umfang des äusseren Bogenganges gebildet. Seine anteroposteriore Länge beträgt $17 \mathrm{~mm}(0,42$ $\mathrm{mm})$ und die dorsoventrale Breite $8 \mathrm{~mm}(0,2 \mathrm{~mm})$.

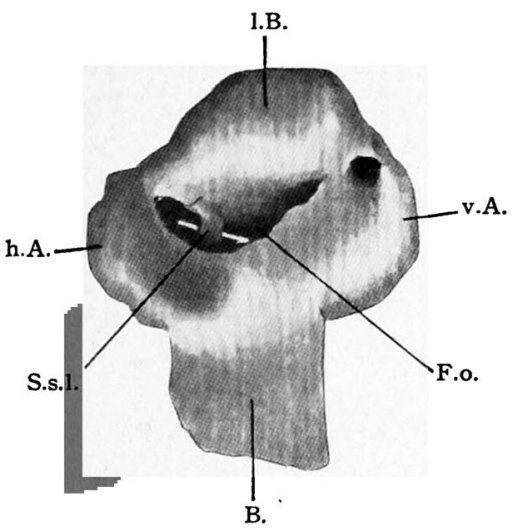

Fig. '.

Iaterale Ansicht desselben Modells. v.A. Vordere Ampulle. h.A. Hintere Ampulle. T.s. Tectum synoticum. B.F.a. Beginn des Foramen acusticum. B.F.e. Beginn des Foramen endolymphaticum. F.o. Foramen ovale. B. Basalplatte. S.s.l. Septum semicirculare laterale. I.B. Iateraler Bogengang. 
Die Ohrkapsel ist niedrig und enthält einen von ohen nach unten abgeplatteten Raum. Auf der oberen Wand befinden sich inmen die bereits schwach differenzierten drei Bogengänge. Das $4 \mathrm{~mm}(0,1 \mathrm{~mm})$ lange Septum semicirculare laterale ist etwas besser ausgebildet als das $2 \mathrm{~mm}(0,05 \mathrm{~mm})$ lange Septum posterius, während das Septum anterius noch nicht entwickelt ist. Die unvollstündig entwickelte vordere und hintere Kuppel ist noch niedrig.

Die Anlage der Ohrkapsel kommt hier als das rerdichtete Kapselgewebe in der Umgebung des häutigen Labyrinthes vor und lässt sich leicht vom gewöhnlichen embryonalen Bindgewebe unterscheiden. Nur der vordere und hintere Teil der medialen Kapselwand bestehen schon aus Vorknorpel. Ventral und medial an dieser Wand liegt das Ganglion acusticum, das kaudal $\mathrm{zu}$ einem langgestreckten Ganglion acusticum poșterius wird. Das ursprüngliche Foramen ovale ist vollständig von der lockeren Bindegewebsschicht ausgefüllt.

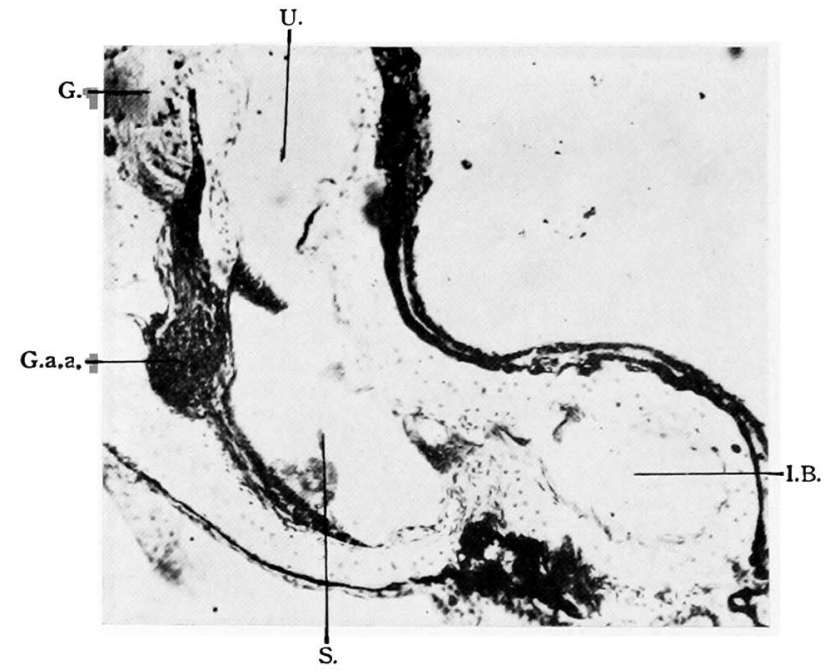

Fig. 3.

Frontaler Durchschnitt des Iabyrinthes. (Stadium 1, rechts, Vergr. $=$ ca. 100 mal, U. Utriculus. S. Sacculus. I.B. I.ateraler Bogengang. (i. Gehirn. G.a.a. Ganglion ac. anterius.
Der Utriculus und Sacculus sind noch nicht vollständig voneinander getrennt, sondern bilden zusarnmen einen grossen gemeinsamen Raum, den Utriculosacculus. Eine grosse Macula acustica liegt am immeren unteren Umfang des Sacculus und trägt ein hohes Epithel. Die Macula utriculi befindet sich am Boden des Recessus utriculi. Hier sind noch die anderen Nervenendstellen in mehrere Abteilungen getremnt, die Cristae acusticae, Macula lagenae und Macula partis basilaris, während die Macula neglecta noch nicht vollständig isoliert ist. Der Ductus endolympaticus ist auch noch nicht sichtbar.

\section{Stadium 2.}


Ganze Länge $17 \mathrm{~mm}$, Stainmlänge $7 \mathrm{~mm}$, Schwanzlä̀nge $10 \mathrm{~mm}$ : Die Augenbläschen sind äusserlich bemerkbar, die Linse ist kuglig und die Retina stark pigmentiert.

Aus dem linken Ohr habe ich ein 40 mal vergrössertes Wachsplattenmodell angefertigt; der anteroposteriore Durchmesser beträgt $46 \mathrm{~mm}$

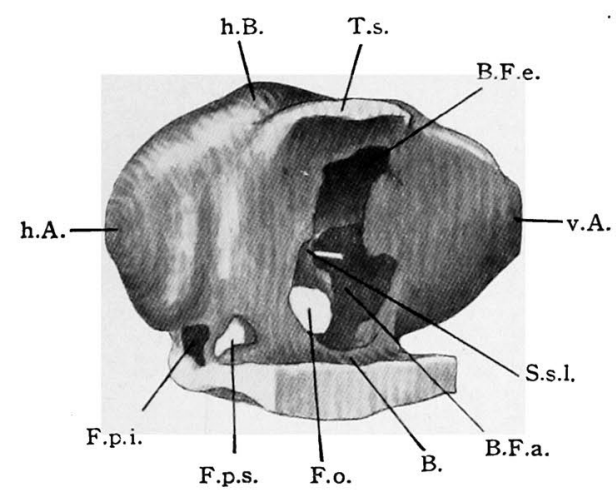

Fig. 4.

Mediale Ansicht des Modells der linken Ohrkapsel. Stadium 2, 40 mal vergrössert modelliert.

flach, aber in ihrem mittleren Teil etwas nach unten konvex. Im lateralen Rande umschliesst sie den lateralen Bogengang. Der mittlere Abschnitt der medialen Kante des Daches entspricht dem Sinus superior utriculi, in dem die vorderen und hinteren Bogengänge zusammentreffen.

Die mediale Wand zeigt schon eine ziemlich gute Ausbildung. Hier befindet sich in der Mitte eine grosse längliche, $20 \mathrm{~mm}(0,5) \mathrm{mm})$ lange, $1 \mathrm{~mm}(0,27 \mathrm{~mm})$ breite Spalte, die von oben weit in den Boden einschneidet. Der obere kleinere Teil dieser Lücke entspricht dem Foramen enido-

$(1,15 \mathrm{~mm})$, der dorsoventrale 27 $\mathrm{mm}(0,67 \mathrm{~mm})$ und der mediolaterale $25 \mathrm{~mm}(0,62 \mathrm{~mm})$. Die hoch gewordene Ohrkapsel ist ziemlich gut ausgebildet; besonders hat die hintere Kuppel an Höhe zugenommen. Der frontale Durchschnitt der vorderen und hinteren Kuppel sieht dreiseitig aus, während der des mittleren Kapselteils unregelmässig-vierseitig ist. Die dorsale Kapselwand ist abgerundet-vierseitig und etwas nach aussen geneigt. Im allgemeinen ist diese Wand

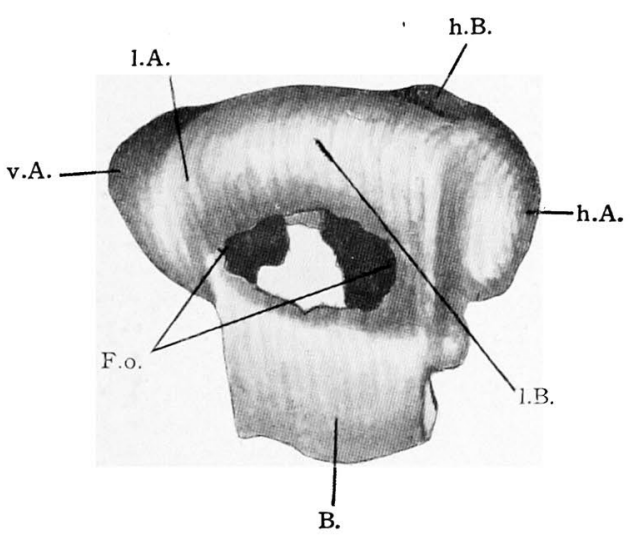

Fig. 5.

Laterale Ansicht desselben Modells. v.A. Vordere Ampulle. h.A. Hintere Ampulle. l.A. Laterale Ampulle. B.F.e. Beginn des Foramen endolymphaticum. B.F.a. Beginn des Foramen acusticum. S.s.l. Septum semicirculare laterale. F.o. Foramen ovale. F.p.s. Foramen perilymphaticum superius. F.p.i. Foramen perilymphaticum inferius. 1.B. Lateraler Bogengang. h.B. Hinterer Bogengang. T.s. Tectum synoticum. B. Basalplatte. 
lymphaticum in späteren Stadien, der untere grössere den Foramina acustica. Hinten dieser Lücke, dicht über dem Boden, liegt das kleine Foramen perilymphaticum superius, $4 \mathrm{~mm}(0,1 \mathrm{~mm})$ lang, $3 \mathrm{~mm}(0,07$ $\mathrm{mm}$ ) breit. Direkt nach hinten davon findet man das kleine Foramen perilymphaticum inferius, $3 \mathrm{~mm}(0,07 \mathrm{~mm})$ lang, $2 \mathrm{~mm}(0,05 \mathrm{~mm})$ breit. Der Ductus perilymphaticus inferius erweitert sich jetzt neben dem VagusGanglion zum Saccus perilymphaticus und steht durch den Canalis perilymphaticus anastomoticus mit dem Lymphraum in Schädel in Verbindung. Die ventrale Kapselwand hat keine bedeutenden Veränderungen erfahren. Die viel deutlicher als vorher entwickelte Lateralwand hat an Höhe zugenommen. Ungefähr in ihrer Mitte findet man in der Nähe des Bodens das horizontal stehende Foramen ovale, das $17 \mathrm{~mm}(0,42 \mathrm{~mm})$ lang und $9 \mathrm{~mm}(0,22 \mathrm{~mm})$ breit ist.

Der Innenraum der Kapsel ist etwas gut ausgebildet; besonders hat der hintere Kuppelraum, wie schon gesagt, eine merkliche Fortbildung erfahren. Die Septa semicircularia sind hesser entwickelt als vorher. Man sieht das Septum semicirculare anterius und laterale, während an der Stelle des Septum posterius nur eine schwache Leiste auf der medialen und dorsalen Ohrkapselwand vorkommt, welche in späteren Stadien noch besser ausgebildet ist. Die Länge des Septum anterius beträgt $4 \mathrm{~mm}$

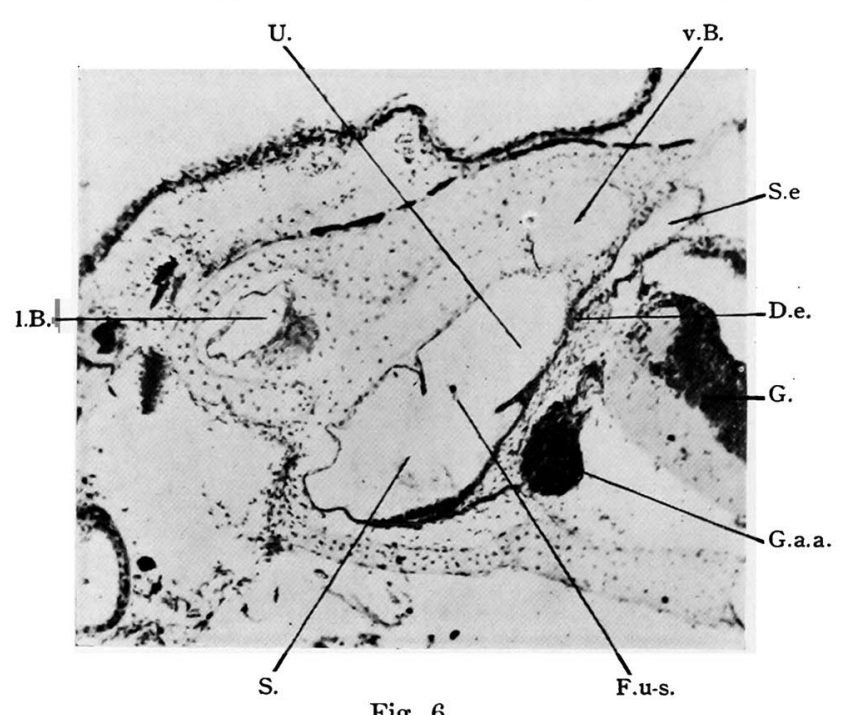

Fig. 6.

Frontaler Durchschnitt des Iabyrinthes. (Stadium 2, links, Vergr. =ca. $60 \mathrm{mal}$ ). G. Gehirn. U. Utriculus. S. Sacculus. v.B. Vorderer Bogengang. 1.B. I.ateraler Bogengang. F.u-s. Foramen utriculosacculare. S.e. Saccus endolymphaticus. D.e. Ductus endolymphaticus. G.a.a. Ganglion ac. anterius.
$(0,1 \mathrm{~mm})$ und die des Septum laterale $3 \mathrm{~mm}(0,07 \mathrm{~mm})$.

In der vorderen Kuppel befindet sich die vordere und laterale Ampulle nebst dem vordersten Teil des die beiden aufnehmenden Recessus utriculi ; in der hinteren dagegen jetzt nur der hintere $\mathrm{Ab}$ schnitt des hinteren Bogenganges mit seiner Ampulle. Der Mittelteil des Kapselraumes nehmen den Utriculus 
Die Ohrkapsel und der schalleitende Apparat von $\mathrm{H}_{y}$ la arborea japonica.

mit Sinus superior utriculi und den Sacculus mit Lagena, Pars basilaris, Pars neglecta sowie den noch nicht vollständig ausgebildeten perilymphatischen Raum auf.

Das Ganglion acusticum zeigt hier keine deutlichere Veränderung als vorher. Von seinem vorderen Teil geht eine feine Nervenfaser zum Sacculus, während der hintere keinen Ast verzweigt. Das Foramen ovale ist auch von der lockeren Bindegewebsschicht wie vorher ausgefüllt; so existiert der schalleitende Apparat noch nicht in diesem Stadium.

Im Vergleich mit dem vorigen Stadium ist das häutige Labyrinth hicr viel besser entwickelt, und an der medialen Sacculuswand ist der Ductus endolymphaticus zu sehen. Dieser mündet an der oberen medialen Sacculuswand, direkt oben vom Foramen utriculosacculare aus, steigt entlang der medialen Wand des Sinus superior auf und erweitert sich bald zum Saccus endolymphaticus. Der Utriculus und Sacculus sind durch das ziemlich grosse Foramen utriculosacculare verbunden. In diesem Stadium trifft man zum ersten Male die Differenzierung von allen Nervenendstellen, die aber noch sehr schwach entwickelt sind. So sieht man hier den ersten Beginn der Pars neglecta mit ihrer Nervenendstelle.

\section{Stadium 3 .}

Ganze Länge $31 \mathrm{~mm}$, Stammlänge $10 \mathrm{~mm}$, Schwanzlänge $21 \mathrm{~mm}$.

Aus dem linken Ohr wurde ein 40 mal vorgrössertes Wachsplattenmodell angefertigt; es besitzt einen anteroposterioren Durchmesser von $65 \mathrm{~mm}(1,02 \mathrm{~mm})$, einen dorsoventralen von $30 \mathrm{~mm}(0,75 \mathrm{~mm})$ und einen mediolateralen von $35 \mathrm{~mm}(0,87 \mathrm{~mm})$. Die mediale Wand der Ohrkapsel steht noch vertikal, von vorn nach hinten sehr verlängert.

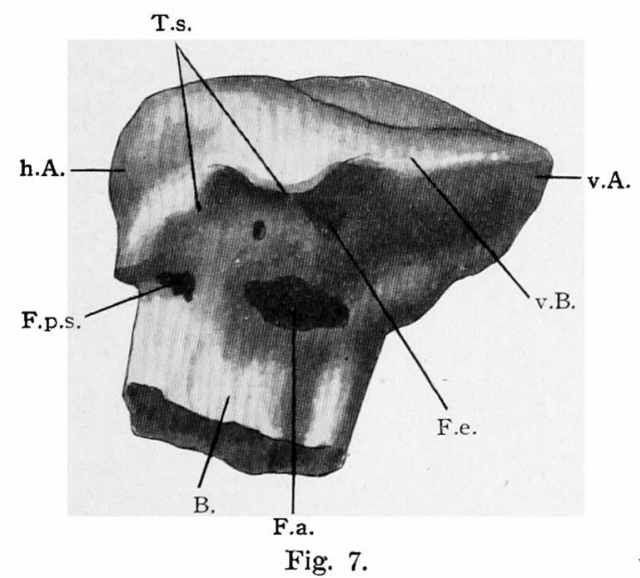

Mediale Ansicht des Modells der linken Ohrkapsel. Stadium 3, 40 mal vergrössert modelliert und auf $2 / 3$ verkleinert reproduziert.
Im Gegensatz zum vorigen Stadium ist die Ohrkapsel jetzt überall gewachsen und einige Foramina sind geformt, wenmgleich die Foramina acustica noch nicht vollständig wie im bleibenden Zustande geteilt sind. Hier befinden sich im ganzen drei Löcher. In mitten ventral nahe dem Boden sieht man eine, von vorn nach hinten liegende, sehr grosse Lücke, die Anlage der Foramina acustica; sie ist $15 \mathrm{~mm}$ $(0,37 \mathrm{~mm})$ lang, $6 \mathrm{~mm}(0,15$ 
$\mathrm{mm}$ ) breit. Weiter hinten davon findet sich auch das von vorn nach hinten gerichtete Foramen perilymphaticum superius nahe dem Boden, das

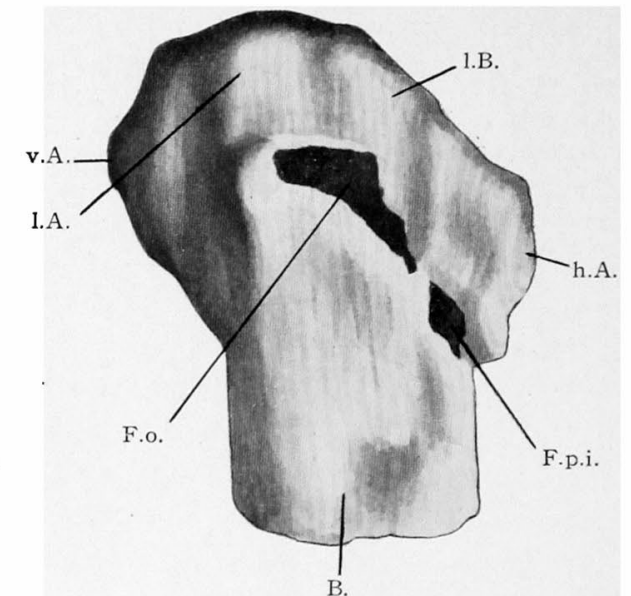

Fig. 8.

Iaterale Ansicht desselben Modells. v.A. Vordere Ampulle. h.A. Hintere Ampulle. 1.A. Laterale Ampulle. F.e. Foramen endolymphaticum. F.a. Foramen acusticum. F.p.s. Foramen perilymphaticum superius. F.p.i. Foramen perilymphaticum inferius. F.o. Foramen ovale. T.s. Tectum synoticum. v.B. Vorderer Bogengang. 1.B. Lateraler Bogengang. B. Basalplatte. $5 \mathrm{~mm}(0,12 \mathrm{~mm})$ lang, $2 \mathrm{~mm}$ $(0,05 \mathrm{~mm})$ breit ist. Oberhalb der Mitte der Wand liegt das kleine, $7 \mathrm{~mm}(0,17 \mathrm{~mm})$ lange und $5 \mathrm{~mm}$ $(0,12 \mathrm{~mm})$ breite, ovale Foramen endolymphaticum. Seine Längsachse ist von hinten oben nach vorn unten gerichtet. Lateral hinten davon findet man das kleine Foramen perilymphaticum inferius, am Boden des Hinterteils der hinteren Kuppel, dicht hinten und etwas unten vom Foramen ovale; es ist $9 \mathrm{~mm}(0,22$ $\mathrm{mm})$ lang, $5 \mathrm{~mm}(0,12 \mathrm{~mm})$ breit. Die dorsale Wand erscheint ungefähr oval. Ihr lateraler Umfang verbindet sich mit der lateralen Kante, die der Prominentia sem. lateralis entspricht und die obere Begrenzung des Foramen ovale darstellt. Die mediale

Kante ist dagegen nicht so einfach gebildet; hier finden sich oberhalb dieses Randes die gut entwickelte, von diesem ausgehende, breite synotische Decke. Die Verbindung des Tectum synoticum mit Ohrkapsel beschränkt sich nicht nur auf der dem Sinus superior entsprechenden Strecke der Kapsel, sondern dehnt sich in Form eines kleinen Fortsatzes auch nach hinten bis zur Gegend des medialen-Umfanges der hinteren Kuppel aus. Von der Mitte dieses Umfanges verläuft die Prominentia semicircularis anterior entlang dem vorderen Rande nach vorn aussen, die Prominentia semicircularis posterior dagegen nach hinten aussen.

Das Foramen ovale der lateralen Kapselwand hat hier nicht nur seine Länge vermehrt, sondern auch seine Lage verändert. Das häutige Labyrinth ist ziemlich gut entwickelt und dementsprechend sind die ventralen und lateralen Partien der Ohrkapsel sehr gut gewachsen. Der äussere Bogengang liegt an der lateralen Kante des Kapseldaches beschränkt und damit findet sich das Foramen ovale zwischen dem freien lateralen Rande der ventralen Wand und seinem unteren Rande. Daher 
kamn man sagen, dass das Foramen ovale in diesem Stadium viel länger als vorher geworden ist; es bildet eine längliche grosse Lücke und ist ziemlich stark von vorn oben nach hinten unten geneigt; es ist $25 \mathrm{~mm}$ $(0,62 \mathrm{~mm})$ lang und $10 \mathrm{~mm}(0,25 \mathrm{~mm})$ breit.

Die ventrale W'and bildet jetzt lateralwärts ziemlich ausgedehnt, die direkte Fortsetzung der Basalplatte und endet aussen mit einem freien Rande als die untere Begrenzung des Foramen ovale.

Der Innenraum der Ohrkapsel, besonders das Cavum cochleare, ist jetzt noch nicht vollständig differenziert. Der längliche Hauptraum ist von vorn nach hinten geneigt. Das Foramen ovale bleibt noch offen. An mittleren unteren Teil der medialen Wand befindet sich eine grosse Lücke, die späteren Foramina acustica. Hinten liegt das kleine Foramen perilymphaticum superius. Medial oben setzt sich der Hauptraum in ein kleines abgeplattetes Cavum sinus superioris fort, in den das Cavum semicirculare anterius und posterius einmündet. In dem Gebiet dieses mittleren oberen Abschnittes, das den Sinus superior enthält, findet sich auch auf der medialen Wand das Foramen endolymphaticum. Das Foramen perilymphaticum superius liegt nahe dem Boden des hinteren Teiles, das Foramen perilymphaticum inferius ausserordentlich weit lateral davon.

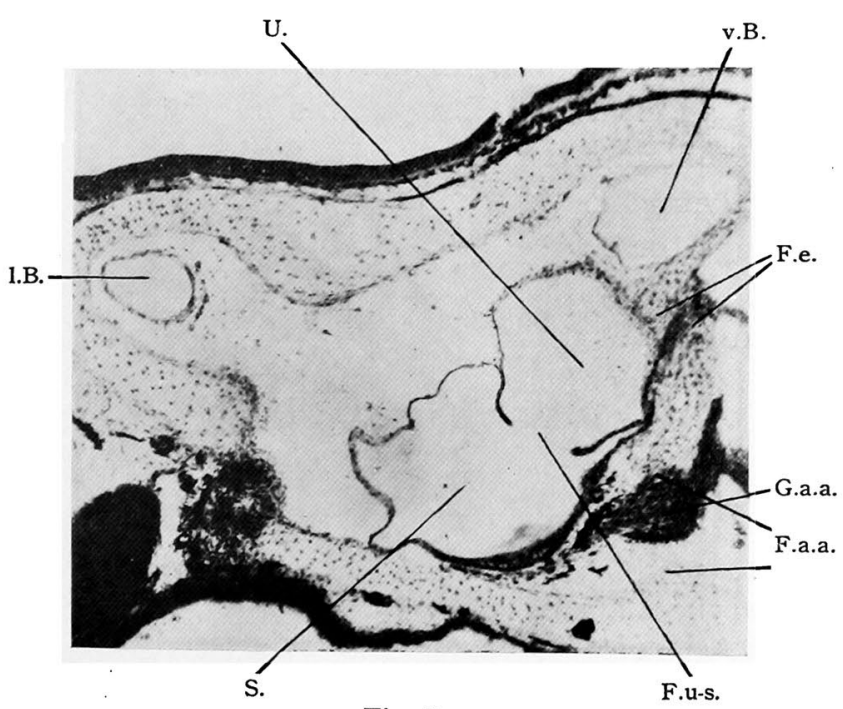

Fig. 9.

Frontaler Durchschnitt des Labyrinthes. (Stadium 3, links, Vergr. $=$ ca. 55 mal). U. Utriculus. S. Sacculus. F.u-s. Foramen utriculosacculare. v.B. Vorderer Bogengang. I.B. Iateraler Bogengang. F.e. Foramen endolymphaticum. F.a.a. Foramen ac. anterius. G.a.a. Ganglion ac. anterius.
Das $6 \mathrm{~mm}(0,15$ $\mathrm{mm}$ ) lange Septum semicirculare laterale erstreckt sich von der Gegend des oberen Teiles der inneren Wand frontal nach unten. Das $10 \mathrm{~mm}(0,25 \mathrm{~mm})$ lange Septum anterius und $7 \mathrm{~mm}$ $(0,17 \mathrm{~mm})$ lange posterius liegen ebenfalls fast horizontal auf der medialen Wand. Der grösste Teil des Hauptraumes enthält den Utriculus, Sacculus, die Lage- 
na, Pars basilaris und Pars neglecta sowie den ziemlich gut entwickelten perilymphatischen Raum.

An der medialen Wand findet sich in seinem mittleren ventralen Teil, am Boden, eine grosse Lücke, durch welche der kleine Acusticus eintritt. Das noch nicht vollständig entwickelte Ganglion acusticum liegt darin. Das Foramen ovale ist von der kernreichen Bindegewebsschicht, die dem Operkulargewebe des schalleitenden Apparates entspricht, verschlossen.

Die drei häutigen Bogengänge sind schon mässig gut entwickelt. Der Utriculus und Sacculus sind miteinandar durch das ziemlich schmale Foramen utriculosacculare begrenzt, und medialwärts oberhalb dieses Foramen mündet der Ductus endolymphaticus in den Sacculus. Auch das Cavum perilymphaticum ist hier ziemlich gut entwickelter und ist im mittleren Abschnitt der Ohrkapsel vom Sacculus, Utriculus und Sinus superior sowie von der Kapselwand umgeben.

4. Stadium 4 .

Ganze Länge $35 \mathrm{~mm}$, Stammlänge $12 \mathrm{~mm}$, Schwanzlänge $23 \mathrm{~mm}$; die beiden hinteren Extremitäten sind noch nicht gut gegliedert.

Das 33 mal vergrösserte Wachsplattenmodell des rechten Ohres trägt die Form einer vierseitigen Pyramide und besitzt einen anteroposterioren Durchmesser von $69 \mathrm{~mm}(2,09 \mathrm{~mm})$, einen dorsoventralen von $46 \mathrm{~mm}$ $(1,39 \mathrm{~mm})$ und einen mediolateralen von $42 \mathrm{~mm}(1,27 \mathrm{~mm})$. Die Ohrkapsel hat an Höhe sehr zugenommen, besonders ihre untere Partie, was mit der weiteren Ausbildung der cochlearen Teile des häutigen Labyrinthes sowie des perilymphatischen Raumes zusammenhängt.

Die Kapseldecke ist ungefähr abgerundet-viereckig. Die Prominentia semicircularis posterior ist viel kräftiger wulstartig als die Prominentia anterior. Die Prominentia semicircularis lateralis ist scharf und kräftig ausgebildet. Der vordere Umfang dieses Wulstes bildet hier mit der lateralen Kapselwand die grosse Prominentia ampullae lateralis. Hier findet sich die kräftige, sagittal an den lateralen Bogengang angesetzte Crista parotica, die in das vordere Ende des Quadratum sich bindegewebig fortsetzt.

Die Form des Tectum synoticum ist durch das Wachstum des Gehirns, das sich weiter nach kaudal ausdehnt, verändert. Es setzt sich nämlich vou der Gegend der Foramina perilymphatica ab in Form einer Leiste auf dem medialen Umfang der hinteren Ohrkapselkuppel fort und steht mit der Crista occipitalis lateralis in Verbindung.

Die grösste mediale Wand ist schon vollständig verschlossen, so dass sie jetzt drei Foramina acustica für die Zweige des Acusticus trägt und 
mässig stark nach innen konvex ist. Ungefähr in ihrer Mitte sieht man die längliche, von vorn nach hinten stehende Prominentia utriculosac-

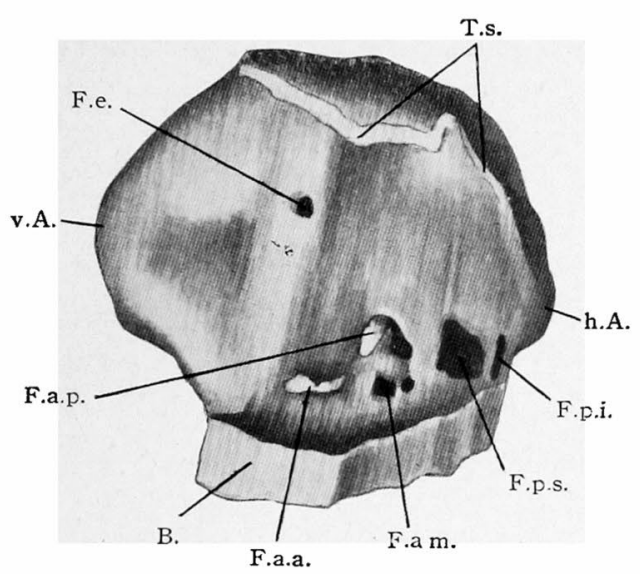

Fig. 10

Mediale Ansicht des Modells der rechten Ohrkapsel. Stadium 4, 33 mal vergrössert modelliert und auf $2 / 3$ verkleinert reproduziert. cularis, die noch schwach entwickelt von der Ungebung nicht scharf abgesetzt ist. Oberhalb der Mitte dieser Prominenz liegt das kleinste Foramen endolymphaticum, das 2 $\mathrm{mm}(0,06 \mathrm{~mm})$ lang, $2 \mathrm{~mm}$ $(0,06 \mathrm{~mm})$ breit ist. Weiter unten davon befinden sich von vorn nach hinten sechs Löcher nahe dem Boden. Das vorderste, kleine, länglich-ovale Foramen acusticum anterius steht von vorn nach hinten neigend und ist $8 \mathrm{~mm}(0,24$ $\mathrm{mm}$ ) laug, $2 \mathrm{~mm}$ (0,06 mm) breit. Hinten davon liegen zwei Foramina, ein oberes grosses Foramen ac. posterius und ein unteres kleines Foramen ac. medium. Das erstere ist $5-5 \mathrm{~mm} \quad(0,15-0,15 \mathrm{~mm})$ und das letztere 4-2 $\mathrm{mm}(0,12-$ $0,06 \mathrm{~mm}$ ) gross. Diese beiden liegen übereinander und sind durch eine senkrechte Knorpelsäule vom Foramen ac. anterius abgetrennt. Der hintere untere Rand dieser Knorpelsäule steht durch die feine Knorpelsubstanz mit der Basalplatte in Verbindung. Die zwischen dem Foramen ac. posterius und medium liegende Knorpelscheidewand bildet einen horizontal von vorn nach hinten liegenden schmalen Knorpelbalken, wodurch

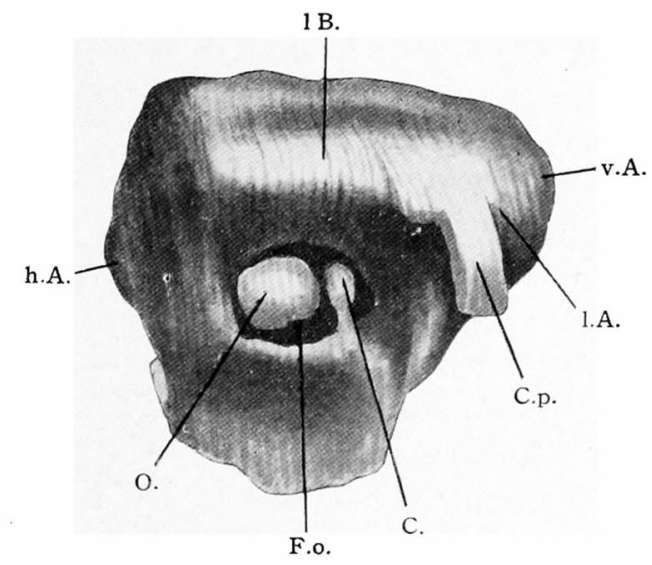

Fig. 11.

Laterale Ansicht desselben Modells. v.A. Vordere Ampulle. h.A. Hintere Ampulle. l.A. Laterale Ampulle. F.e. Foramen endolymphaticum. F.a.a. Foramen acusticum anterius. F.a.m. Foramen acusticum medium. F.a.p. Foramen acusticum posterius. F.p.s. Foramen perilymphaticum superius. F.p.i. Foramen perilsmphaticum inferius. F.o. Foramen ovale. T.s. Tectum synoticum. I.B. Lateraler Bogengang. C.p. Crista parotica. O. Operculum. C. Columella. B. Basalplatte. 
sie beide voneinander vollständig abgetrennt sind. Daraus kann man den Abtrenuungsvorgang des Foramen ac. medium so verstehen, dass das bisherige einfache grosse Foramen acusticum zuerst in zwei vordere und hintere Löcher geteilt wird und dam das letztere wieder durch einen horizontalen Knorpelbalken in das Foramen posterius und medium vollkommen abgetrennt wird. Direkt hinten vom Foramen medium, dicht oben vom Boden, findet sich das grösste Foramen perilymphaticum superius, das seine Längsachse von vorn oben nach hinten unten richtet und $8 \mathrm{~mm}(0,24 \mathrm{~mm})$ lang, $6 \mathrm{~mm}(0,18 \mathrm{~mm})$ breit ist. Hinten und aussen davon, zugleich dicht über dem Boden liegt das länglich-ovale, $9 \mathrm{~mm}(0,27 \mathrm{~mm})$ lange und $5 \mathrm{~mm}(0,15 \mathrm{~mm})$ breite Foramen perilymphaticum inferius, das mit seiner Spitze nach oben gerichtet steht. Jie mediale Wand geht nach unten ungefähr senkrecht in den Ohrkapselboden über.

Die ventrale Wand geht nach vorn beinahe horizontal von ihrer Mitte allmählich in die vordere Kuppel über, hinten dagegen fast rechtwinklig in die hintere.

Auf der lateralen Wand steht das Foramen ovale durch den schalleitenden Apparat geschlossen und zwischen diesen beiden besteht eine durch das Bindegewebe ausgefüllte, grosse ringförmige Lücke; es ist $21 \mathrm{~mm}(0,63 \mathrm{~mm})$ lang and $14 \mathrm{~mm}(0,42 \mathrm{~mm})$ breit. Die Bildung des schalleitenden Apparates begimnt zuerst in diesem Stadium, aber noch nicht vollständig. Das Operculum stellt eine länglich-runde Platte dar, die ungefähr vertikal steht und jetzt den hinteren grossen Abschnitt des Foramen ovale rerschliesst; es ist $12 \mathrm{~mm}(0,39 \mathrm{~mm})$ lang, $10 \mathrm{~mm}(0,3$ $\mathrm{mm}$ ) breit. Ausserdem findet sich im vorderen Teile des Foramen ein kleiner Fortsatz, die Columellaanlage. Er ist jedoch noch nicht vollkommen entwickelt und ragt mit einem kleinen Stiel vom vorderen ventralen Rande des Foramen ovale hervor; seine ganze Länge beträgt $10 \mathrm{~mm}(0,3 \mathrm{~mm})$ und die Breite $5 \mathrm{~mm}(0,15 \mathrm{~mm})$.

Der Innenraum der Ohrkapsel ist sehr weit entwikelt und lässt sich im allgemeinen in zwei ungleiche Teile unterscheiden. Man sieht die drei Septa semicircularia; die Länge des Septum anterius beträgt $14 \mathrm{~mm}$ $(0,42 \mathrm{~mm})$, die des Septum posterius $9 \mathrm{~mm}(0,27 \mathrm{~mm})$ und die des Septum laterale $13 \mathrm{~mm}(0,39 \mathrm{~mm})$.

Der Mittelteil der medialen Wand des Cavum vestibulare buchtet sich medialwärts aus. Diese Ausbuchtung, der Recessus utriculosaccularis, steht von vorn nach hinten und geht allmählich in den vorderen und hinteren Teil der medialen Wand über.

Das Cavum cochleare ist im Gegensatz zum Cavum vestibulare ein- 
fach, geht vorn ohne merkliche Grenze in das Cavum ampullae anterioris über und endet hinten blind, während sein lateraler Teil direkt rom schalleitenden Apparat verschlossen wird.

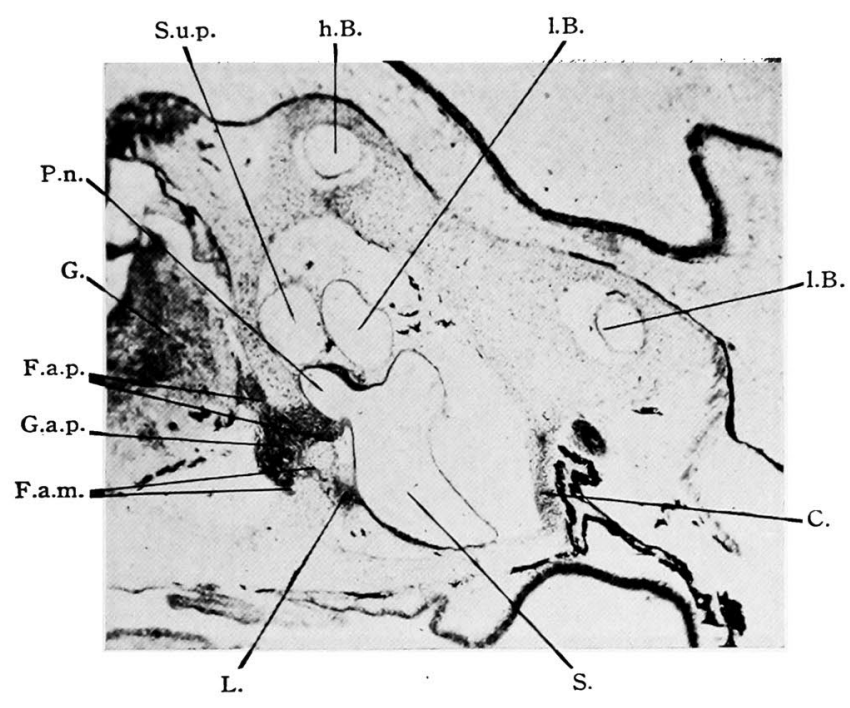

Fig. 12.

Frontaler Durchschnitt des Labyrinthes. (Stadium 4, rechts, Vergr. =ca. 35 mal). S.u.p. Sinus utriculi posterior. I.B. I.ateraler Bogengang. h.B. Hinterer Bogengang. S. Sacculus. L. Lagena. F.a.m. Foramen ac. medium. F.a.p. Foramen ac. posterius. P.n. Pars neglecta. G.a.p. Ganglion ac. posterius. G. Gehirn. C. Columella.

Das Ganglion acusticum liegt in einer Nische der medialen Wand nahe dem Boden der Schädelhöhle ; vorn von ihm tritt der Ramus anterior, nach hinten der Ramus medius sowie posterior aus. Der grosse Ramus anterior, der in die Ramuli rec. utric., sace., amp. ant., und amp. lat. zerfällt, und der grösste Ramus posterior, der sich in die Ramuli neglect. basilaris, und amp. post. verzweigt, ge-

hen zum Labyrinth; der vom letzteren verzweigte Ramus medius gelangt durch das Foramen ac. medium zur Lagena.

Das Operkulargewebe ist hier deutlich entwickelt und stellt eine ziemlich dicke vorknorpelige Platte dar. Die aus dem unteren vorderen Rande des Foramen ovale hervorgehende Columella lässt sich fast durch alle 5 Schnitte (30 $\mu$ weit) der Serie hindurch verfolgen, und ihre hintere Seite ist mit dem vorderen Rande des Operculum durch das Bindegewebe verbunden. Der schalleitende Apparat steht mit den Rande des Foramen eine kurze Strecke weit bindegewebig in Verbindung.

Das häutige Labyrinth ist bedeutend gewachsen. Der Utriculus und Sacculus sind durch das enge Foramen utriculosacculare kommuniziert. Lateral vom Sacculus befindet sich jetzt das grosse Cavum perilymphaticum.

\section{Stadium 5 .}

Ganze Länge $45 \mathrm{~mm}$, Stammlänge $20 \mathrm{~mm}$, Schwanzlänge $25 \mathrm{~mm}$; 
die beiden hinteren, etwa $16 \mathrm{~mm}$ langen Extremitäten sind gut gegliedert, die vorderen $5 \mathrm{~mm}$ lang.

Aus dem rechten Ohr wurde ein 33 mal vergrössertes Wachsplattenmodell angefertigt. Diese knorpelige Ohrkapsel ist viel deutlicher als beim vorigen Stadium und fast vollständig entwickelt. Der Sagittaldurchmesser des Modells ist länger und der dorsoventrale kürzer geworden ; der erstere misst $85 \mathrm{~mm}(2,57 \mathrm{~mm})$, der letztere $56 \mathrm{~mm}(1,69 \mathrm{~mm})$ und der mediolaterale $55 \mathrm{~mm}(1,66 \mathrm{~mm})$.

Das Dach der Kapsel steht beinahe horizontal, etwas nach aussen hinten geneigt. An seinem vorderen medialen Rande beginnt die Prominentia semicircularis anterior, die von der vorderen Ampulla nach hinten innen zieht. Kaudal liegt die Prominentia semicircularis posterior ; die Grenze zwischen den beiden ist hier auch nicht deutlich. Die laterale Wand bildet gleichzeitig den oberen Rand der lateralen Kapselwand und geht rückwärts in die deutlich vorspringende Prominentia semicircularis lateralis über, die ungefähr in seiner Mitte liegt und unten den oberen Rand des Foramen ovale bildet.

Das Tectum synoticum ist hier gut verknorpelt und verbindet sich nach vorn mit dem oberen inneren Rande der vorderen Kuppel, hinten dagegen setzt es sich ventralwärts in die Crista occipitalis lateralis, am inneren Umfang der hinteren Kuppel, fort und läuft auf den Occipitalbogen aus, die kaudale Wand der Schädelhöhle bildend. Daher besteht das Tectum nicht mehr aus einer sagittalen Platte allein, sondern man kam drei Teile unterscheiden, nämlich zwei ungefähr sagittal stehende Leisten, die vordere und mittlere Randleiste, und eine hintere, fast froutal

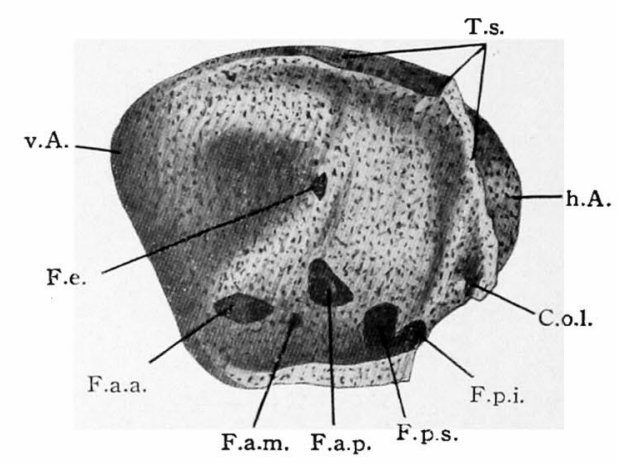

Fig. 13.

Mediale Ansicht des Modells der rechten Ohrkapsel. Stadium 5, 33 mal vergrössert modelliert und auf $1 / 2$ verkleinert reproduziert. stehende Platte.

Die mediale Wand steht ungefähr vertikal. An ihrem Mittelteil findet man die grosse wulstartige Prominentia utriculosaccularis, die jetzt stark nach medial vorspringt. Oberhalb ihrer Mitte bemerkt man das kleine dreieckige $7 \mathrm{~mm}(0,21$ $\mathrm{mm})$ lange, $4 \mathrm{~mm}(0,12 \mathrm{~mm})$ breite Foramen endolymphaticum, das mit seiner Spitze nach hinten oben gerichtet steht. Weiter unten davon liegen hintereinander fünf Foramina. 
Das vorderste, länglich-ovale Foramen acusticum anterius ist $11 \mathrm{~mm}$ $(0,33 \mathrm{~mm})$ lang, $6 \mathrm{~mm}(0,18 \mathrm{~mm})$ breit; seine Längsachse ist von vorn

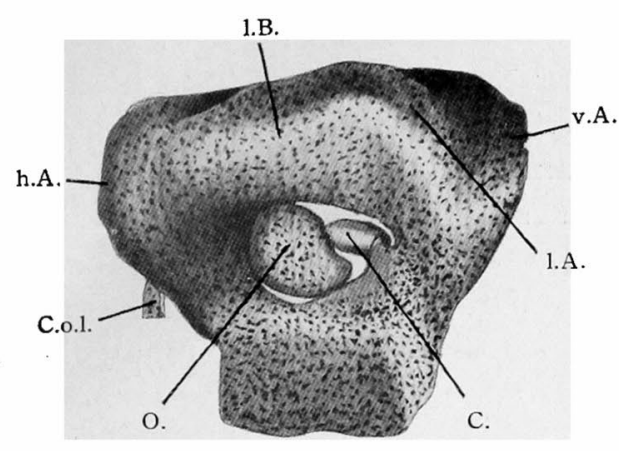

Fig. 14 .

Laterale Ansicht desselben Modells. v.A. Vordere Ampulle. h.A. Hintere Ampulle. 1.A. Laterale Ampulle. F.e. Foramen endolymphaticum. F.a.a. Foramen acusticum anterius. F.a.m. Foramen acusticum medium. F.a.p. Foramen acusticum posterius. F.p.s. Foramen perilymphaticum superius. F.p.i. Foramen perilymphaticum inferius. T.s. Tectum synoticum. C.o.l. Crista occipitalis lateralis. l.B. Iateraler Bogengang. O. Operculum. C. Columella. nach hinten gerichtet. Direkt hinten unten davon sieht man das kleinste unregelmässige Foramen acusticum medium, das eine Länge von $5 \mathrm{~mm}(0,15 \mathrm{~mm})$ und eine Breite von $4 \mathrm{~mm}(0,12$ $\mathrm{mm}$ ) besitzt. Etwas weiter hinten oben von ihm findet sich das grössere, unregelmässig-rundliche Foramen acusticum posterius, das $9 \mathrm{~mm}(0,27 \mathrm{~mm})$ lang, $7 \mathrm{~mm}(0,21 \mathrm{~mm})$ breit ist. Weiter hinten unten davon sieht man das Foramen perilymphaticum superius und inferius, die beide miteinander kommunizieren. Der Durchmesser des ersteren misst 13-9 mm $(0,39$ $0,27 \mathrm{~mm})$, der des letzteren $10-9$ $\mathrm{mm}(0,3-0,27 \mathrm{~mm})$.

Die ventrale, ungefähr horizontale Wand ist einfach. Ihr mittlerer lateraler Randteil ist leicht gewölbt und zieht etwas nach oben, besonders der orale Abschnitt ragt ziemlich deutlich hervor und umgibt das Foramen ovale. Im Vergleich mit vorigem Stadium ist das Foramen ovale viel grösser und von dem besser entwickelten schalleitenden Apparat verschlossen. Das Foramen ist fast oval, seine Ebene steht ungefähr senkrecht, die Längsachse aber etwas von vorn lateral nach hinten medial gerichtet; es ist $31 \mathrm{~mm}(0,93 \mathrm{~mm})$ lang, $20 \mathrm{~mm}(0,6 \mathrm{~mm})$ breit. Das knorpelige Operculum stellt eine unregelmässig-ovale Platte dar, die mit seiner stumpfen Spitze nach vorn unten, etwas innen, geneigt steht und $19 \mathrm{~mm}(0,57 \mathrm{~mm})$ lang, $17 \mathrm{~mm}(0,5 \mathrm{~mm})$ breit ist. Die Lücke zwischen den beiden ist viel enger geworden als vorher. Die Columella ist jetzt noch nicht verknorpelt und ragt mit einem schmalen Stiel vom vorderen unteren Rande des Foramen ovale in die Lücke hervor. Die Columella liegt im vorderen oberen Abschnitt des Foramen als ein dreieckiges Plättchen, das ungefähr horizontal von vorn nach hinten gerichtet steht und mit seinem hinteren spitzen Ende dicht dem vorderen oberen Rande des Operculum anliegt; sie ist $13 \mathrm{~mm}(0,39 \mathrm{~mm})$ lang, $11 \mathrm{~mm}(0,32 \mathrm{~mm})$ 
breit. Ausserdem gehen vom vorderen Rande der Columella zwei kleine Fortsätze aus und endigen frei nach vorn in den vorderen Umfang des Foramen ovale.

Die mediale Wand des Cavum vestibulare reicht nach vorn bis zum Recessus ampullae anterioris und ihr vorderer oberer Teil wird vom Septum semicirculare anterioris gebildet. Sie steht frontal, doch ziemlich stark nach aussen geneigt und ist $19 \mathrm{~mm}(0,57 \mathrm{~mm})$ lang. Ebenso nach hinten wird sie auch vom Septum semicirculare posterius gebildet. Dieses liegt ungefähr horizontal, steigt etwas nach vorn auf und ist zwischen der medialen Wand des Carum vestibulare und der Mitte der hinteren Kapseldecke ausgespannt; es ist $15 \mathrm{~mm}(0,45 \mathrm{~mm})$ lang. Zwischen den beiden freien Rändern dieser Septa hindurch führt das Foramen pro sinu superior vom Cavum vestibulare in den Recessus sinus superioris. Der Recessus utriculosaccularis wölbt sich nach aussen als die Prominentia recessus utriculosaccularis vor und ist gegen den vor ihm gelegenen Recessus ampullae anterioris undeutlich abgesetzt. Unter diesem Recessus liegen die drei Foramina acustica und zwei Foramina perilymphatica auf medialer Wand. Diese Foramina münden ungefähr in der Grenzlinie des Cavum vestibulare und cochleare ein.

Die Decke wird lateral vom Septum semicirculare laterale gebildet, das immer senkrecht steht und $25 \mathrm{~mm}(0,75 \mathrm{~mm})$ lang ist. Es hört nach hinten mit einem, nach medial konkaven Rande auf, der oben in das Septum semicirculare posterius, unten gegen die untere Umrandung des Recessus ampullae posterioris verläuft. Die Mündung des lateralen Bogenganges liegt ungefähr etwas vorn, in der Mitte der lateralen Wand, nahe dem weiten Recessus ampullae lateralis.

Auf der lateralen Wand des Cavum cochleare liegt das Foramen ovale, das durch den schalleitenden Apparat geschlossen ist. Der Boden des Carum ist etwas konkav, die hintere Wand zeigt eine kleine Erhebung, die ihn vom Cavum restibulare abgrenzt. Dagegen findet man nach oral bis zum Recessus ampullae anterioris keine deutliche Leiste; so steigt der vordere Teil des Carum nach vorn allmählich auf und geht in das Cavum ampullae anterioris über. Seine mediale konvexe Seite ist mit dem unteren Teil der medialen Wand des Cavum vestibulare verschmolzen.

Die Bildung des Knorpels geschieht gleichzeitig an allen Stellen der Kapsel, während sie sich nicht gleichmässig von den Zentren über die ganze Kapsel ausbreitet. Hier entsteht noch nicht der echte Knorpel, sondern die Zellen sind nur an manchen Stellen grösser und blasig geworden. Auf den Schnitten der dorsolateralen Kapselwand finden 
sich mehrere grossblasige Knorpelzellen, vor allem im lateralen Abschnitt des lateralen Bogenganges, ebenso in allen, von hier ausstrahlenden

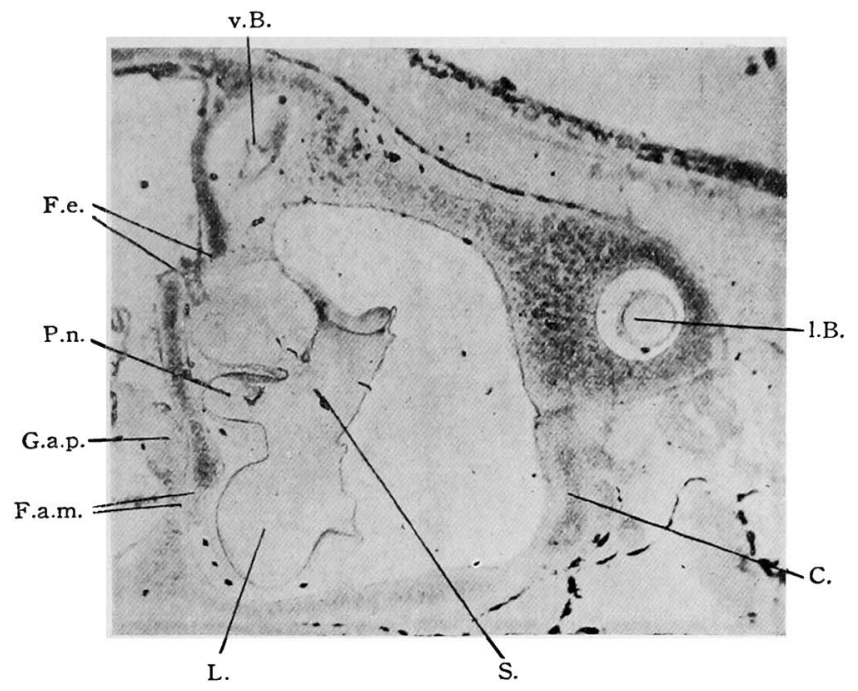

Fig. 15 .

Frontaler Durchschnitt des Labyrinthes. (Stadium 5, rechts, Vergr.=ca. 35 mal). S. Sacculus. I. Jagena. P.n. Pars neglecta. $\quad$. B. Vorderer Bogengang. 1.B. Lateraler Bogengang. F.a.m. Foramen ac. medium. F.e. Foramen endolymphaticum. C. Columella. G.a.p. Ganglion ac. posterius.
Stellen, während in der Mittelpartie der medialen Kapselwand nur die jungen kleinen Zellen zu sehen sind. Die laterale Wand trägt schon den K.norpel, die Columella besitzt dagegen noch keine Knorpelzellen. Die ventrale Wand hat ebenso dort, wo sie sich der Basalplatte nähert, viele grossblasige Zellen, so dass diese Stelle und der oben beschriebene laterale Teil des late-

ralen Bogenganges den deutlichsten Fortschritt der Knorpelsubstanz zeigen. Der Vorknorpel des an hinterer medialer Wand ansetzenden Tectum synoticum ist schon vollkommen verknorpelt.

6. Stadium 6 .

Exemplar gleich nach der Metamorphose, $15 \mathrm{~mm}$ Stammlänge.

Aus dem rechten $\mathrm{Ohr}$ wurde ein 33 mal vergrössertes Wachsplattenmodell angefertigt; es besitzt einen anteroposterioren Durchmesser von $85 \mathrm{~mm}(2,57 \mathrm{~mm})$, einen dorsoventralen von $53 \mathrm{~mm}(1,6 \mathrm{~mm})$ und einen mediolateralen ron $58 \mathrm{~mm}(1,75 \mathrm{~mm})$.

Die knorpelige Ohrkapsel ist jetzt fast vollständig ausgebildet, besonders hat der kaudoventrale Teil ihrer Medialwand eine wesentliche Entwicklung erfahren; die zwei Foramina perilymphatica sind fast vollständig entstanden und voneinander gut $z u$ unterscheiden. Die Ohrkapsel hat in Bezug auf das Formverhältnis schon ihren maximalen Entwicklungsgrad erreicht und nimmt im weiteren Entwicklungsverlaufe nur an Dicke und Grösse zu, ohne ihre Gestalt zu verändern. Die drei Foramina acustica, ein Foramen endolymphaticum sind gut ausgebildet. 
Das kleinste Foramen endolymphaticum ist länglich-viereckig, $4 \mathrm{~mm}$ $(0,12 \mathrm{~mm})$ lang, $2 \mathrm{~mm}(0,06 \mathrm{~mm})$ breit und steht mit seiner Spitze nach

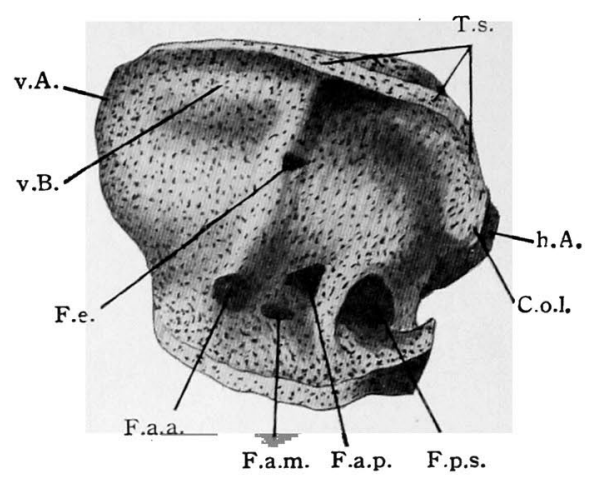

Fig. 16.

Mediale Ansicht des Modells der rechten Ohrkapsel. Stadium 6, 33 mal vergrössert modelliert und auf $x / 2$ verkleinert reproduziert. hinten gerichtet. Das grössere, $9 \mathrm{~mm}(0,27 \mathrm{~mm})$ lange und 7 $\mathrm{mm}(0,21 \mathrm{~mm})$ breite Foramen acusticum anterius ist länglichrund und steht mit seiner Längsachse etwas von linten unten nach vorn oben geneigt. Das kleinere, horizontale Foramen acusticum medium ist länglichoval und $6 \mathrm{~mm}(0,18 \mathrm{~mm})$ lang, $3 \mathrm{~mm}(0,09 \mathrm{~mm})$ breit. Das grössere, dreieckige Foramen acusticum posterius ist $8 \mathrm{~mm}$ $(0,24 \mathrm{~mm})$ lang, $7 \mathrm{~mm}(0,21 \mathrm{~mm}$ breit und steht mit seiner einen Spitze nach vorn gerichtet. Das grösste Foramen perilymphaticum superius ist unregelmässig-rund und $11 \mathrm{~mm}(0,33 \mathrm{~mm})$ lang, $10 \mathrm{~mm}(0,3 \mathrm{~mm})$ breit; das Foramen inferius dagegen rundlich und $8 \mathrm{~mm}(0,24 \mathrm{~mm})$ lang, $8 \mathrm{~mm}(0,24 \mathrm{~mm})$ breit.

Die Decke der Kapsel ist ungefähr rundlich-viereckig und ziemlich stark nach aussen geneigt. Das Foramen ovale ist länglich-oval und wird durch den schon stark entwickelten schalleitenden Apparat geschlossen ; seine Längsachse ist fast horizontal von vorn lateral nach hinten medial gerichtet; es ist $35 \mathrm{~mm}(1,06 \mathrm{~mm})$ lang, $19 \mathrm{~mm}$ $(0,57 \mathrm{~mm})$ breit. Das Operculum ist eine unregelmässige, sagittal stehende Platte, die mit seiner Spitze nach vorn unten, etwas innen gerichtet steht und $18 \mathrm{~mm}(0,54 \mathrm{~mm})$ lang, $20 \mathrm{~mm}$ $(0,6 \mathrm{~mm})$ breit ist; es liegt ungefähr vertikal im hinteren

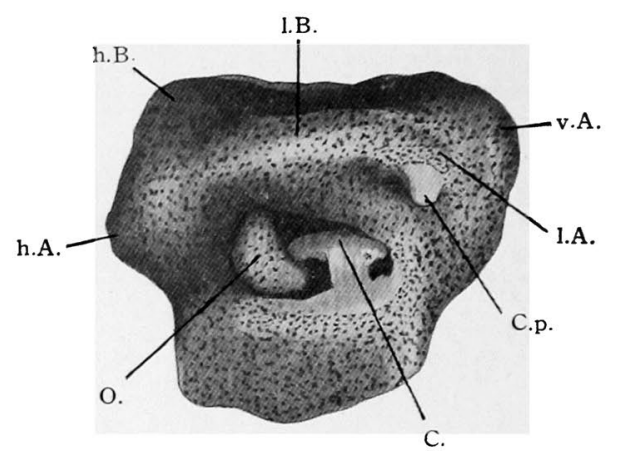

Fig. 16.

Laterale Ansicht desselben Modells. v... Vordere Ampulle. h.A. Hintere Ampulle. l.A. Laterale Ampulle. v.B. Vorderer Bogen. gang. l.B. I ateraler Bogengang. h.B. Hinterer Bogengang. F.e. Foramen endolỵmphaticum, F.a.a. Foramen acusticum anterius. F.a.m. Foramen acusticum medium. F.a.p. Foramen acusticum posterius. F.p.s. Foramen perilymphaticum superius. T.s. Tectum synoticum. C.o.l. Crista occipitalis lateralis. C.p. ('rista parotica. O. Operculum. C. Columella. 
Abschnitt des Foramen ovale und ist durch die sehr schmale Lücke ron knorpeliger Ohrkapselwand vollkommen abgetrennt. Die Columella ist noch nicht ganz verknorpelt und stellt eine horizontal liegende, $18 \mathrm{~mm}$ $(0,54 \mathrm{~mm})$ lange, $14 \mathrm{~mm}(0,12 \mathrm{~mm})$ breite Platte dar, die mit einem stark nach medial konkaven, $4 \mathrm{~mm}(0,12 \mathrm{~mm})$ breiten Stiel vom ventralen vorderen Rande des Foramen ovale in die Fenestra von lateral hineinragt und sich nach hinten bis zum vorderen mittleren Rande des Operculum erstreckt. An seinem vorderen lateralen Rande befestigt sich ein kleiner Fortsatz, der oralwärts gerichtet vom vorderen Umfang des Foramen ovale hervorragt; er ist $5 \mathrm{~mm}(0,15 \mathrm{~mm})$ lang, $5 \mathrm{~mm} .(0,15 \mathrm{~mm})$ breit. Ausserdem findet man auch den oben erwähnten Fortsatz, der nach vorn innen gerichtet steht.

Der Innenraum der Kapsel zeigt hier keinen bedeutend'n Unterschied vom vorigen Stadium. Die Länge des Septum semicirculare anterius beträgt $27 \mathrm{~mm}(0,81 \mathrm{~mm})$, die des Septum posterius $14 \mathrm{~mm}$ $(0,42 \mathrm{~mm})$ und die des Septum laterale $25 \mathrm{~mm}(0,75 \mathrm{~mm})$.

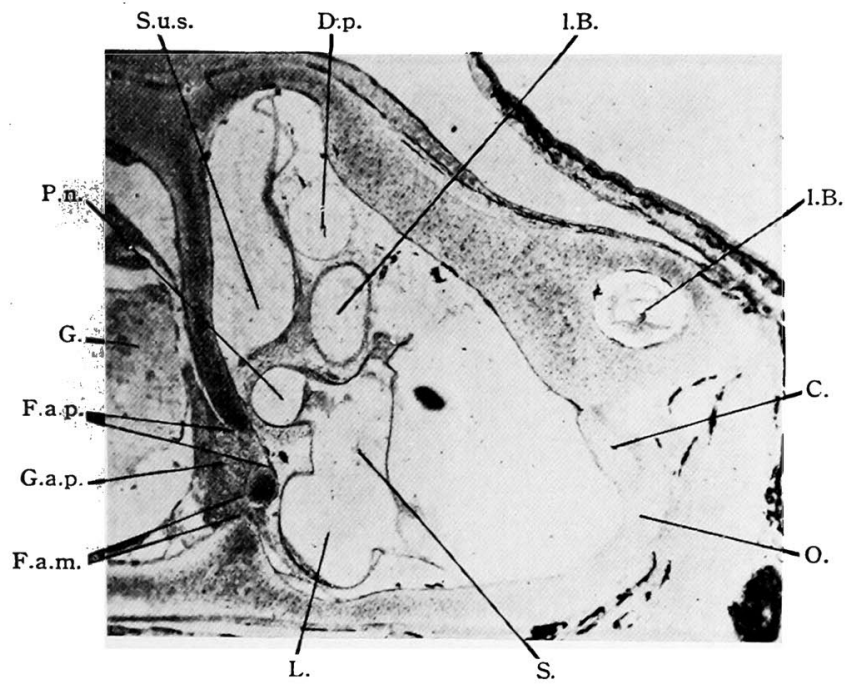

Fig. 18.

Frontaler Durchschnitt des I abyrinthes, (Stadium 6, rechts, Vergr. = ca. "35 mal). G. (iehirn. S.u.s. Sinus utriculi superior. S. Sacculus. I. Lagena. P.n. Pars neglecta. I.B. Lateraler Bogengang. I.p. Ductus perilymphaticus. F.a.p. Foramen ac. posterius. F.a.m. Foramen ac. medium. G.a.p. Ganglion ac. posterius. O. Operculum. C. Columella.
- Im V'ergleich mit dem vorigen Stadium ist die Kuorpelbildung der Ohrkapsel jetzt sehr vorgeschritten zu sehen und es finden sich auch noch in der medialen Wand viel vermehrte Knorpelelemente. Im hinteren Abschnitt des Foramen ovale ist das Operculum verknorpelt, während die Columella noch nicht knorpelig ist und nur aus einer Lage von rundlichen Vorknorpelzellen besteht. Auf den Schnitten durch den ventralen Rand des Foramen ovale finden sich ebenso noch vorknorpelige kleine Zellen, besonders in dessen 
vorderen Teil, während im vorderen, hinteren und oberen Rande desselben viele junge Knorpelzellen zu sehen sind. Bei keinem Stadium konnte ich ein im ventralen Umfang völlig knorpelig geschlossenes Foramen ovale feststellen.

\section{Stadium 7 .}

Ausgewachsenes Exemplar, $30 \mathrm{~mm}$ Stammlänge.

Aus dem rechten Ohr wurde ein 33 mal vergrössertes Wachsplattenmodell angefertigt. Sein anteroposteriorer Durchmesser beträgt $105 \mathrm{~mm}$ $(3,18 \mathrm{~mm})$, der dorsoventrale $56 \mathrm{~mm}(1,69 \mathrm{~mm})$ und der mediolaterale $76 \mathrm{~mm}(2,3 \mathrm{~mm})$. Die Ohrkapsel ist hier schon zum Teil verknöchert, während ihr grösserer Teil noch knorpelig ist. Es ist bemerkenswert, dass die Kapsel an Höhe relativ klein, das Wachstum der ventralen

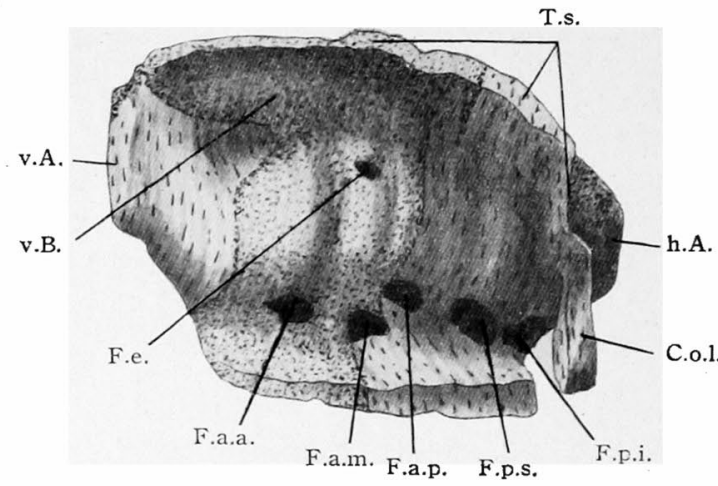

Fig. 19.

Mediale Insicht des Modells der rechten Ohrkapsel.

Stadium 7,33 mal vergrössert modelliert und auf $1 / 2$ verkleinert reproduziert. Partien der Ohrkapsel bedeutend ist.

Das Kapselclach ist, wie beim vorigen Stadium, ziemlich stark nach aussen und etwas nach hinten geneigt und besteht zum grossen Teil aus Knorpelmasse, während ihr hinterer medialer Abschnitt schon verknöchert ist. Die durch die drei Bogengänge verursachten WV̈lste sind scharf und kräftig ausgeprägt. Im Vergleich

mit vorigem Stadium ist hier die Crista parotica viel bedeutender entwickelt und sagittal mit seinem kräftigen dicken Rande vom laterooralen Rande des Kapseldaches nach lateral ausgewachsen.

Die mediale Wand wird auch aus Knochen und Knorpel zusammengesetzt, so dass der vordere und hintere grössere Abschnitt ganz aus

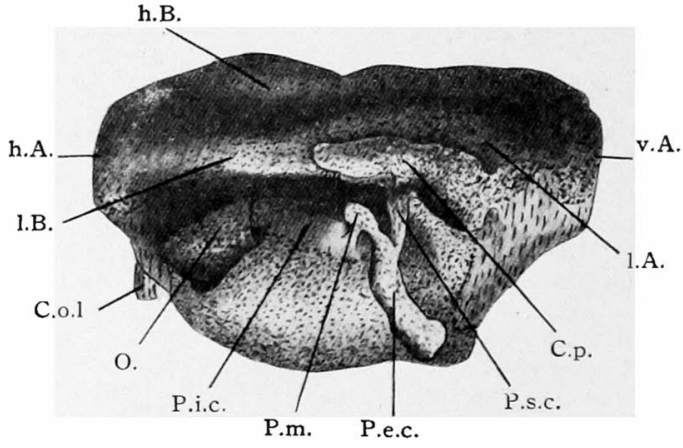

Fig. 20

I.aterale Ansicht desselben Modells. 
Knochenmasse besteht und zwischen den beiden ein sehr breites Knorpelband liegt; das letztere zieht vom mittleren oberen Abschnitt der medialen

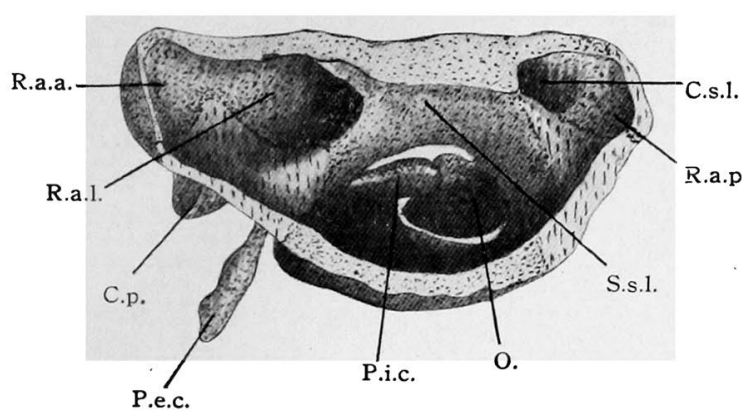

Fig. 21.

Sagittalschnitt durch dasselbe Modell, laterale Hälfte von medial gesehen. v.A. Vordere Ampulle. h.A. Hintere Ampulle. l.A. Jaterale Ampulle. F.e. Foramen endolymphaticum. F.a.a. Foramen acusticum anterius. F.a.m. Foramen acusticum medium. F.a.p. Foramen acusticum posterius. F.p.s. Foramen perilymphaticum superius. F.p.i. Foramen perilymphaticum inferius. T.s. Tectum synoticum. C.o.l. Crista occipitalis lateralis. v.B. Vorderer Bogengang. h.B. Hinterer Bogengang. l.B. Lateraler Bogengang. C.p. Crista parotica, O. Operculum. P.e.c. Pars externa columellae. P.m. Pars media. P.i.c. Pars interna columellae. P.s.c. Processus superior columellae. C.s.l. Canalis semicircularis lateralis. S.s.l. Septum semicirculare laterale. R.a.a. Recessus ampullae anterioris. R.a.p. Recessus ampullae posterioris. R.a.l. Recessus ampullae lateralis.
Wand nach dorsal, weiter bis zum vorderen medialen Rande des Daches hin und verbreitet sich im ganzen Teil des vorderen Bogenganges. Das kleinste Foramen endolymphaticum liegt im oberen mittleren Knorpelteil und ist $3-4 \mathrm{~mm} \quad(0,09-0,12$ $\mathrm{mm})$ gross. In der vorderen ventralen Knorpelmasse findet sich das länglich-ovale Foramen acusticum anterius, das mit seiner Längsachse von vorn nach hinten gerichtet steht und $11 \mathrm{~mm}(0,33$ $\mathrm{mm})$ lang, $7 \mathrm{~mm} \quad(0,21$ $\mathrm{mm})$ breit ist. Das dreieckige, $8 \mathrm{~mm} \quad(0,24$ $\mathrm{mm})$ lange, $5 \mathrm{~mm} \quad(0,15$

$\mathrm{mm}$ ) breite Foramen acusticum medium ist etwas grösser als das Foramen posterius und befindet sich teils vorn und oben im Knorpel- und teils unten im Knochenteil ; seine Spitze ist nach hinten gerichtet. Das kleine Foramen acusticum posterius ist oval und liegt in der Knorpelsubstanz, mit Längsachse etwas von vorn oben nach hinten unten stehend ; es ist $7 \mathrm{~mm}(0,21 \mathrm{~mm})$ lang, $5 \mathrm{~mm}(0,15 \mathrm{~mm})$ breit. Hinten von ihm, auch im Knochenteil sieht man das rundlich-viereckige Foramen perilymphaticum superius, das mit seiner Längsachse von vorn oben nach hinten unten geneigt steht und $10 \mathrm{~mm}(0,3 \mathrm{~mm})$ lang, $8 \mathrm{~mm}(0,24 \mathrm{~mm})$ breit ist. Das grösste Foramen perilymphaticum inferius ist oval, findet sich in der hinteren medialen Wand der Kapsel und ist $12 \mathrm{~mm}(0,36$ $\mathrm{mm}$ ) lang, $8 \mathrm{~mm}(0,24 \mathrm{~mm})$ breit; seine Längsachse steht von medial nach lateral gerichtet. Der breite Boden der Kapsel liegt beinahe horizontal und sein lateraler Rand ist stark nach aufwärts gekrümmt.

Das Knorpelband der medialen Wand verbreitet sich auch auf der vorspringenden ventralen. Wand der Pars cochlearis und zieht in zwei 
vordere und hintere Schenkel aus, von denen der eine nach Crista parotica hin zieht, der andere dagegen nach oben auf den hinteren Randteil

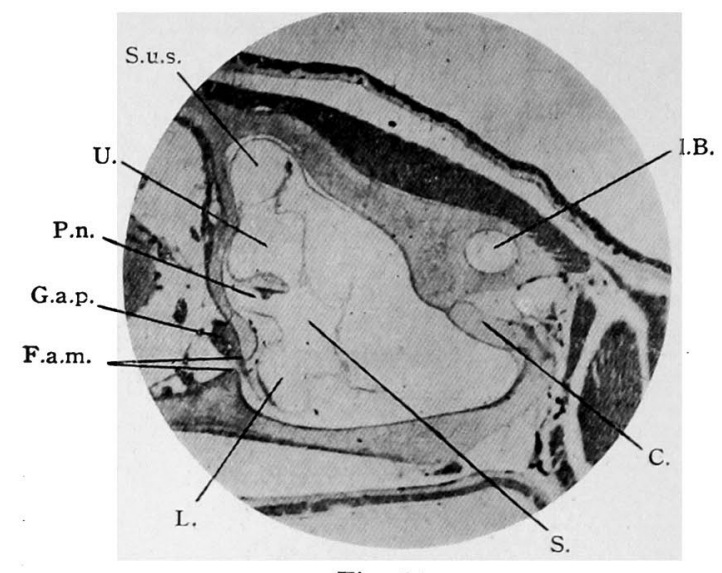

Fig. 2\%.

Frontaler Durchschnitt des Labyrinthes. (Stadium 7 . rechts, Vergr. $=$ ca. $15 \mathrm{mal})$. U. Utriculus. S. Sacculus. I. Lagena. P.n. Pars neglecta. v.B. Vorderer Bogengang. 1.B. Lateraler Bogengang. F.a.m. Foramen ac. medium. G.a.p. Ganglion ac. posterius. C. Columella.

des Foramen ovale verläuft, um in den hinteren Abschnitt des hinteren Bogenganges überzugehen.

Im hinteren Abschnitt der lateralen Kapselwand befindet sich das Foramen ovale, das laterokaudodorsalwärts offen steht. Es ist $25 \mathrm{~mm}(0,75 \mathrm{~mm})$ lang, 45 min $(0,36 \mathrm{~mm})$ breit und jetzt fast vollständig vorn von Columella und gleich hinten von Operculum geschlossen, während in seinem oberen und unteren Abschnitt noch eine kleine Lücke vorhanden ist. Die wichtigste V'eränderung hat

das hinter der Columella gelegene Operculum erfahren. Dieses stellt ein langgestrecktes, halbkugliges. Gebilde dar, das von vorn gesehen fast oval erscheint und mit seiner Spitze der Basis nach vorn aussen gerichtet steht. Mit medialem Rande der Basis ist das Operculum fast rollständig mit dem linteren Rande des Foramen ovale knorpelig verwachsen; der abgerundete untere Rand erreicht dagegen nicht den Unterrand des Foramen, vielmehr bleibt eine

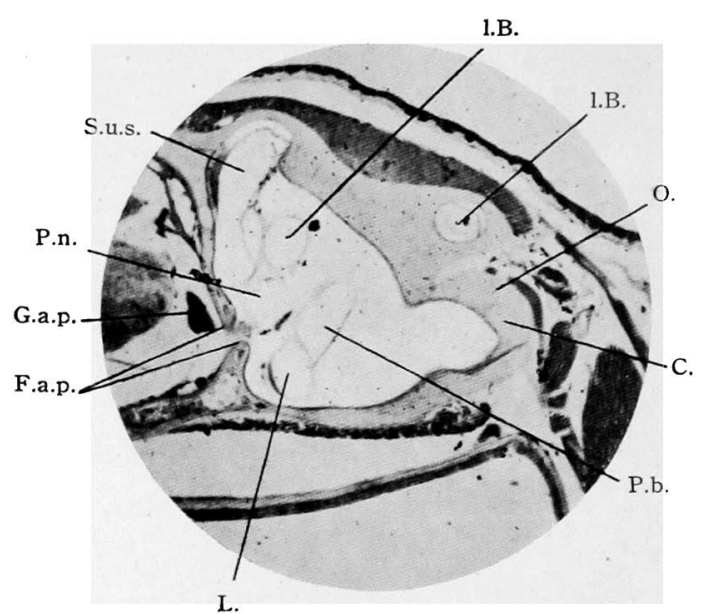

Fig. 23.

Frontaler Durchschnitt des I abyrinthes. (Stadium 7 , rechts, Vergr. =ca. 15 mal). S.u.s. Sinus utriculi superior. P.n. Pars neglecta. I. I.agena. P.b. Pars basilaris. F.a.p. Foramen ac. posterius. (i.a.p. Ganglion ac. posterius. 1.B. Iateraler Bogengang. O. Operculum. C. Columella. 
schmale Lücke dazwischen. Daher kann man sagen, dass das Operculum erst bei jüngeren Exemplaren aus einem selbständig, in dem Foramen ovale vorhandenen Operkulargewebe entsteht und vollkommen von der Ohrkapsel abgetrennt ist, während es beim erwachsenen mit dem hinteren

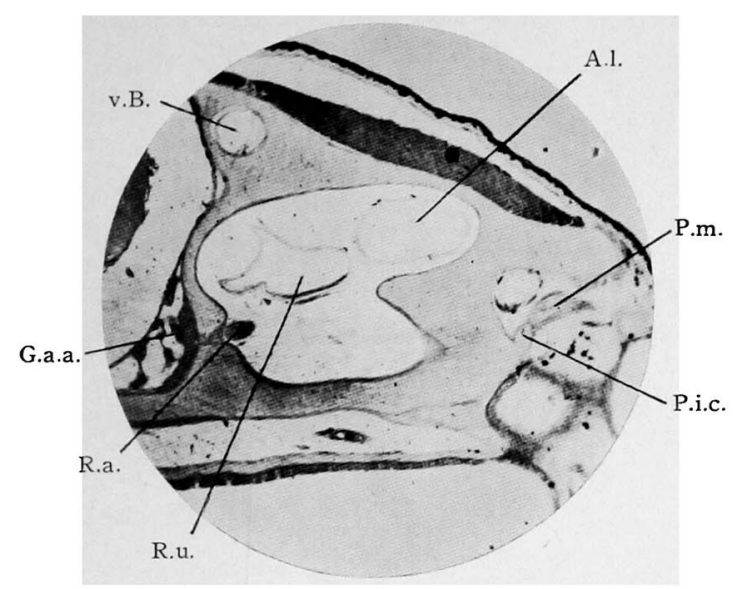

Fig. 24.

Frontaler Durchschnitt des J.abyrinthes. (Stadium 7, rechts, Vergr. =ca. 15 mal). R.u. Recessus utriculi. A.l. Ampulla lateralis. v.B. Vorderer Bogengang. G.a.a. Ganglion ac. anterius. R.a. Ramus anterior.

P.i.c. Pars interna columellae. P.m. Pars media.

ten Platte umgewandelt, ragt als ein schon vollständig entstandener innerer Abschnitt der Columella direkt vom ventralen vorderen Rande des Foramen ovale nach medial hervor und steht horizontal von vorn nach hinten gerichtet. Das hintere Ende dieser Platte erstreckt sich zur konkaven Innenseite des Operculum, mit dem es immer knorpelig verbunden ist; ihr vorderes Ende verbreitet sich dagegen zum vorderen Rande des Foramen und
Rande des Foramen knorpelig sich verbindet.

In diesem Stadium ist die Columella plötzlich sehr stark entwickelt und schon völlig verknorpelt. Sie stellt eine sehr lange, mehrfach geknickte Platte dar, die sich von Ohrkapsel nach aussen bis zum Trommelfell erstreckt und sich jetzt in drei $\mathrm{Ab}$ schnitte einteilen lässt. Die Pars interna columellae ist beim vorigen Stadium noch nicht völlig gebildet, aber jetzt ist sie zu einer ziemlich dicken und brei-

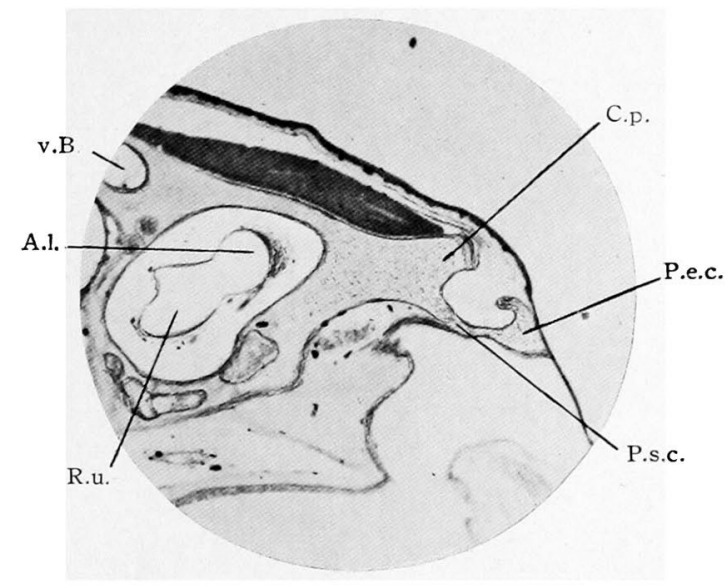

Fig. 25.

Frontaler Durchschnitt des Labyrinthes. (Stadium 7, rechts, Vergr. =ca. 15 mal). R.u. Recessus utriculi. A.l. Ampulla lateralis. v.B. Vorderer Bogengang. C.p. Crista parotica. P.s.c. Processus superior columellae. P.e.c. Pars externa columellae. 
verwächst mit diesem Rande innig; sie ist $30 \mathrm{~mm}(0,9 \mathrm{~mm})$ lang, $16 \mathrm{~mm}$ (0,48 min) breit. Die Pars media bildet die direkte Fortsetzung des vorderen lateralen Randes der Pars interna columellae, sieht erst nach aussen oben und geht damn etwa in rechtwinklicher Knickung in die Pars externa über; sie ist $20 \mathrm{~mm}(0,6 \mathrm{~mm})$ lang. Die Pars extema stellt einen nach vorn angeschwollenen länglichen Stab dar, steht lateroventrooralwärts gerichtet und ist mit dem Trommelfell in Verbindung; ihr Stumpfende ist $25 \mathrm{~mm}(0,75 \mathrm{~mm})$ lang, $10 \mathrm{~mm}(0,3 \mathrm{~mm})$ breit. Sie hat auch einen $15 \mathrm{~mm}(0,45 \mathrm{~mm})$ langen knorpeligen Fortsatz, den Processus superior columellae, der vom vorderen medialen Umfang ihres oberen Abschnittes nach mediodorsal zieht und in die Crista parotica übergeht.

Im Innern der Ohrkapsel befinden sich die knorpeligen Septa semicircularia; nur das Septum anterius ist in seinem vorderen inneren Abschnitt verkuöchert. Seine Länge beträgt $28 \mathrm{~mm}(0,84 \mathrm{~mm})$, die des Septum posterius $15 \mathrm{~mm}(0,45 \mathrm{~mm})$ und die des Septum laterale $23 \mathrm{~mm}$ $(0,69 \mathrm{~mm})$.

\section{Vergleichung und Zusammenfassung.}

\section{Ohrkapsel.}

Die Knorpelbildung der Ohrkapsel bei den Anuren ist schon von Goette ('75) eingehend beschrieben worden. Er sagt bei Unke : ,Während dieser Entwicklung der hinteren Schädelbasis entsteht rund um jedes Gehörorgan eine knorpelige Kapsel, deren innerer unterer Rand mit der Schädelbasis verschmilzt. Dadurch kann leicht der Eindruck hervorgerufen werden, als sei wenigstens die horizontale, die Gehörorgane tragende Platte jener Knorpelkapsel als unmittelbare Fortsetzung der knorpeligen Schädelbasis aus dieser hervorgewachsen. Einer solchen Auffassung widerspricht einfach der Umstand, dass die das Gehörbläschen überziehende Knorpellage am äusseren Umfange bereits entstanden ist, bevor der mittlere Teil der Schädelbasis auch nur angelegt ist, und dass nach innen fortwachsend erst nachträglich mit dem Seitenrande desselben zusammenstösst." Stöhr ('80-'81) beschrieb auch bei den Amphibien, dass die Knorpelbildung am lateralen Teil des lateralen Bogenganges beginnt und sich von hier weiter nach allen Seiten ausbreitet, wobei die mediale Wand zuletzt gebildet wird. Die laterale Wand verschmilzt mit dem aus der Basalplatte hervorgehenden Knorpel, so dass anfänglich eine grosse Lücke, die aber später verschlossen wird, zwischen diesen beiden besteht. Er beschreibt beim Triton noch: ,, Der Raum zwischen diesem sanduhrförmigen Achsenteil und der unteren medialen Kante der 
Ohrkapsel ist durch Knorpel ausgefüllt, so dass eine direkte knorpelige Verbindung zwischen Ohrkapsel und Achsenteil der Basilarplatte besteht. Diese verbindende Knorpelmasse, welche also den peripheren Teil der Basalplatte darstellt, ist nicht durch Wachstum des Achsenteils in die Breite, sondern durch knorpelige Differenzierung des da zwischenliegenden Gewebes von den Olırkapseln aus entstanden." Ganz ähnlich schildert Gaupp ('93) bei den Anuren, sich Stöhr anschliessend. Er sagt bei Rana fusca: ,,Vom Parachordalknorpel (mesotischem Knorpel Stöhrs) erfolgt die Umschliessung der medialventralen Teile des mittleren Labyrinth-Abschnittes (vorzüglich des Sacculus nebst seinen Ausstülpungen, Lagena, P. basilaris und neglecta, sowie zum Teil die Umschliessung des Acusticus). Der ,,periotische“ Knorpel breitet sich von seiner Ausgangsstelle am äusseren Bogengange derart weiter aus, dass er zunächst nach vorn und hinten, immer dem äusseren Bogengange folgend, vorschreitet und für dessen äusseren, oberen und unteren Umfang eine knorpelige Umwandung schafft. Auf den häutigen Canalis ant. und post. übergreifend biegt er vorn und hinten nach innen um, bildet dadurch je einen kuppelförmigen Abschluss der gesamten Kapsel-Anlage und verbindet sich mit dem Parachordale an zwei Stellen."

Nach Peter ('98) geht die Knorpelbildung der Kapsel bei Ichthyophis fast ebenso wie bei den Urodelen vor sich. Ähnlich entsteht nach Gaupp ('90) der Knorpel der Kapsel auch bei den Sauriern. Die Knorpelbildung geht ebenfalls, wie bei den Urodelen, von einem lateral oben befindlichen äusseren Umfang des lateralen Bogenganges und einem medial unten parachordal gelegenen Zentrum aus. Er sagt auch bei den Amnioten: ,Das Skelett zeigt eine entsprechende Umgestaltung. Sie lässt sich, glaube ich, nicht anders auffassen und ausdrücken als durch den Satz: das Skelettmaterial, das bei den Reptilien den vordersten lateralen Abschnitt der soliden „Basalplatte“ bildet, wird bei den Säugern zur Umwandung der Pars cochlearis der Ohrkapsel verwendet. Damit würden also die Begriffe ,Basalplatte“ und „,Ohrkapsel“ nur noch eine formale Bedeutung besitzen. Die mit diesen Namen belegten Bildungen bei verschiedenen Wirbeltieren würden nur insofern untereinander vergleichbar sein, als sie ähnlich geformte Abschnitte des Craniums darstellen, wären aber nicht in Bezug auf das Material, aus dem sie entstehen, als untereinander gleichwertig zu betrachten." Hafferl ('21) sagt beim Gecko, dass die Verknöcherung perichordal in einem direkt neben der Chorda und einem ganz lateral gelegenen Bezirk beginnt, so dass zwischen beiden eine Partie von Knorpel übrig bleibt, die sich bei fortschreitender Verknöcherung $\mathrm{zu}$ einer Fuge verschmälert. Auch 
andere Autoren wie Okajima ('12) bei Hynobius und Stadtmüller ('25) bei Salamandra maculosa sagen, dass die Knorpelbildung der Kapsel am lateralen Teil des lateralen Bogenganges beginnt und sich von hier nach allen Seiten verbreitet, wobei die mediale Wand zuletzt ausgebildet wird.

Beim Stadium 1 (10 mm Gesamtlänge) meines Tieres ist die Cochlea etwas deutlich.abgegrenzt und die drei Bogengangstaschen sind sichtbar. Als Anlage der Ohrkapsel findet man eine schwache Gewebsverdichtung am dorsolateralen Umfang des häutigen Labyrinthes. Sie erstreckt sich von hier eine Strecke weit nach dorsal und lateral, ohne aber den mittleren Abschnitt der medialen Wand zu erreichen; sie dehnt sich nach vorn und binten hin aus, so dass die ganze dorsale Wand uud die vordere und hintere Kuppel schon von der Vorknorpelanlage gebildet werden. Ein zweiter Herd bildet sich ventral in der Ungebung der Cochlea, der durch die Commisura basicapsularis mit dem lateralen Abschnitt der Basalplatte zusammenhängt. Also in früherer Zeit sind zwei Zentren, ein oberes laterales und ein unteres mediales, vorhanden. Beim Stadium 2 (17 mm Gesamtlänge) kommt die Ausbildung des dichten Kapselgewebes weit im dorsolateralen Teil vor und breitet sich von dort wach allen Seiten hin aus. Im allgemeinen beginnt die Bildung des Vorknorpels im oberen lateralen Abschnitt des lateralen Bogenganges und schreitet von hier aus auf die dorsale, laterale, vordere und hintere Kapselwand hin, wobei die mediale Wand zuletzt ausgebildet wird. Bei diesem Stadium hat die Medialwand an ihrem mittleren Teil eine grosse Lücke und zwei Foramina perilymphatica, die beide schon in hinteren unteren Abschnitt vollkommen voneinander abgetrennt sind. Beim Stadium 3 (31 mm Gesamtlänge) ist die Medialwand plötzlich stark entwickelt und zeigt eine grosse Lücke für den Ductus endolymphaticus. In diesem Stadium erscheint jener Teil der medialen Wand, der den Acusticus umgibt und seine Foramina umrandet. Hier ist eine weite Öffnung vorhanden, in dem die Äste des Acusticus mit seinem Ganglion liegen. Beim Stadium 4 (35 mm Gesamtlänge) ist die grosse Lücke der medialen Wand vollkommen von dichtem Kapselgewebe geschlossen und hier sind im ganzen drei Foramina acustica, das Foramen acusticum anterius, posterius und medium für den Ramus medius zur Lagena gebildet. Solchen Zustand sieht man bei vielen ausgewachsenen Urodelen wie Siredon, Triton, Megalobatrachus, Onychodactylus, Hynobius und Salamandra, was schon von Stöhr ('80), Windischman ('81), Retzius ('81), Okajima ('12) u. a. beschrieben worden ist. Von einigen Autoren, Ogushi ('11) bei Trionyx japonicus, Nick ('12) bei Dermochelys, 
Die Ohrkapsel und der schalleitende Apparat von Hyla arborea japonica.

Hafferl. ('21) bei Gecko und mị ('27) bei Clemmys japonica sowie Eumeces latiscutatus, sind die mebrfachen Foramina acustica media auf der medialen Ohrkapselwand gefunden worden.

Dass das Foramen acusticurn medium vom Foramen posterius durch den Knorpel abgeschieden wird, beschreibt schon Gaupp ('00) bei den Amnioten: ,, Die bei den Säugern erfolgende Zerlegung des unteren (des primären hinteren) Foramina in drei Nerveu-Austrittsgebiete, Foramen singulare (R. ampullae posterioris), Area vestibularis inferior. (R. saccularis) und Tractus spiralis foraminosus ( $R$. cochlearis) ist ein sekundärer Vorgang, der mit der schärferen, räumlichen Trennung der bezüglichen Nervenendstellen zusammenhängt, und zudem eine Teilerscheinung der auch an der gauzen übrigen Ohrkapsel zu beobachtenden stärkeren Konsolidierung darstellt." Okajima ('12) sagt beim Hynobius : ,, Ich hatte beim erwachsenen Hynobius gesehen, dass ausser diesen beiden Löchern, Foramen acusticum anterius und posterius für die betreffenden Zweige des Hörnerven, noch eine Anzahl von anderen feinen Löchern (3 bis 6) vorhanden sind, welche für den Sacculus bestimmte feine Nervenäste durchgehen lassen. Es ist wohl $\mathrm{zu}$ vermuten, dass das einfache weite Foramen posterius bei weiterer Entwioklung durch feine Knorpelbalken in komplizierte Fächer abgeteilt wird." Bei Hyla arborea entsteht das Foramen posterius zuerst bei der Larve von $35 \mathrm{~mm}$ Gesamtlänge und zugleich ist das Foramen medium schon gut ausgebildet. Diese beiden Foramina liegen übereinander an der medialen Ohrkapselwand und sind durch eine senkrechte Knorpelsäule vom Foramen anterius abgetrennt. Der hintere untere Rand der letzteren steht durch die feine Knorpelsubstanz mit der Basalplatte in Verbindung. Die zwischen dem Foramen posterius und medium liegende Knorpelscheidewand stellt einen horizontal von vorn nach hinten ziehenden schmalen Knorpelbalken dar. Aus obigen Befunden kann ich mit Sicherheit sagen, dass das grosse einfache Foramen acusticum zuerst in zwei Löcher, ein vorderes und hinteres, geteilt und dann das letztere weiter durch den Knorpelbalken in zwei Foramina, das Foramen posterius und medium, vollständig abgeschieden wird. Beim Stadium 5 (45 mm Gesamtlänge) kommen der echte Knorpel weit am dorsolateralen Teil der Kapsel vor, besonders am lateralen Abschnitt des lateralen Bogenganges und davon nach allen Seiten hin, während in der mittleren Partie der medialen Wand nur die jüngsten Knorpelzellen zu sehen sind. Die ventrale Wand ebenso trägt dort, wo sie sich der Basalplatte nähert, den echten Knorpel. So kann man sagen, dass die Bildung des Knorpels nicht gleichmässig an allen Stellen der Kapsel fortschreitet, sondern sich ungleichmässig von 
einigen Zentren über die ganze Kapsel ausbreitet. In diesem Stadium sind die drei Foramina acustica und das Foramen endolymphaticum schon ziemlich gut gebildet, während die zwei Foramina perilymphatica noch nicht knorpelig voneinander abgetrennt sind. Beim Stadium 6 (15 mm Stammlänge, gleich nach der Metamorphose) ist die Kapsel schon gut verknorpelt und zeigt mit der fertigen Ausbildung der medialen Wand auch die vollständige Abteilung des Foramen perilymphaticum. Die Foramina acustica und Foramina perilymphatica liegen hintereinander in der Nähe des Bodens, ähnlich wie bei den Urodelen, nicht aber gauz primitiv, d. h. die Öffnungen stehen nicht mehr genau hintereinander, sondern das Foramen posterius ist nach dorsal verschoben, so dass dieses oben und hinten vom Foramen nedium liegt. Beim Stadium 7 (30 mm Stammlänge, ausgewachsenes Tier) ist die Kapsel schon zum Teil ziemlich gut verknöchert, während sein grösserer Teil noch ganz kuorpelig ist. Die mediale Wand wird aus Knochen sowie Knorpel zusammengesetzt, so dass ihr vorderer kleiner und hinterer grösserer Abschnitt ganz aus Knochenmasse besteht; zwischen den beiden Massen liegt ein sehr breites Knorpelband; dieses zieht sich von dem mittleren oberen Abschnitt der medialen Wand dorsalwärts weiter zum vorderen medialen Rand des Kapseldaches hin und verbreitet sich im gauzen Teil des vorderen Bogenganges. Das Foramen endolymphaticum und Foramen acusticum anterius liegen in der Knorpelsubstanz, während das Foramen medium teils vorn oben im Knorpel und teils unten in der Knochenwand sich befindet. Ausserdem findet man auch an der medialen Wand noch drei Öffnungen, das Foramen acusticum posterius und Foramen perilymphaticum superius sowie inferius, welche alle hinten ganz im Knochen der Medialwand liegen.

\section{Septa semicircularia.}

Im Innern der Ohrkapsel bilden sich die knorpeligen Septa, welche die Räume für die Bogengänge gegen das Cavum vestibulare commune abgrenzen. Beim Stadium 1 (10 mm Gesantlänge) befindet sich nur das noch nicht knorpelig differenzierte Septum posterius und laterale, während das Septum anterius noch nicht entwickelt ist. Die Länge des Septum posterius beträgt $2 \mathrm{~mm}(0,05 \mathrm{~mm})$ und die des Septum laterale $4 \mathrm{~mm}(0,1 \mathrm{~mm})$. Beim Stadiuın $2(17 \mathrm{~mm}$ Gesamtlänge $)$ findet man zwei Septa semicircularia für den vorderen und lateralen Bogengang, während der Raum für den hinteren Bogengang aber nur durch eine niedrige Leiste ganz unvollkommen gebildet ist. Der vordere und laterale Bogengang haben eine etwas gut entwickelte Spange, das $4 \mathrm{~mm}$ 
$(0,1 \mathrm{~mm})$ lange Septurn anterius und das $3 \mathrm{~mm}(0,07 \mathrm{~mm})$ lange Septum laterale. Beim Stadium 3 (31 $\mathrm{mm}$ Gesamtlänge) sieht man auch ausser dem Septum posterius das etwas stärker entwickelte $10 \mathrm{~mm}(0,25 \mathrm{~mm})$ lange Septum anterius und das $6 \mathrm{~mm}(0,15 \mathrm{~mm})$ lange Septum laterale ; der Raum für den hinteren Bogengang ist jetzt durch eine fast horizontal liegende, $7 \mathrm{~mm}(0,17 \mathrm{~mm})$ lange Spange vollständig abgetrennt. Beim Stadium 4 (35 mm Gesamtlänge) sind die Septa semicircularia mehr und mehr entwickelt, das Septum anterius am besten, und man kann hier die vollständig geschlossene mediale Wand der Kapsel wahrnehmen. Die Länge des Septum anterius beträgt $14 \mathrm{~mm}(0,42 \mathrm{~mm})$, die des Septum posterius $9 \mathrm{~mm}(0,27 \mathrm{~mm})$ und die des Septum laterale $13 \mathrm{~mm}(0,39 \mathrm{~mm})$. Beim Stadium 5 (45 mm Gesamtlänge) sieht man erst alle Septa semicircularia als knorpelige kräftige Spangen, und das Septum laterale ist viel weiter entwickelt als beim vorigen Stadium und besitzt eine Länge von $25 \mathrm{~mm}(0,75 \mathrm{~mm})$. Das Septum anterius ist $19 \mathrm{~mm}(0,57 \mathrm{~mm})$, das Septum posterius $15 \mathrm{~mm}(0,45 \mathrm{~mm})$ lang. Beim Stadium $6(15 \mathrm{~mm}$ Stammlänge, gleich nach der Metamorphose) ist das $27 \mathrm{~mm}(0,8 \mathrm{~mm})$ lange Septum anterius viel stärker ausgebildet, das den Raum für den vorderen Bogengang weit nach oralwärts vom Cavum vestibulare abtrennt. Das Septum posterius ist $14 \mathrm{~mm}(0,42 \mathrm{~mm})$ und das Septum laterale $25 \mathrm{~mm}(0,75 \mathrm{~mm})$ lang. Zuletzt im Stadium 7 (30 mm Stammlänge, ausgewachsenes Tier) ist das Septum anterius nur in seinem vorderen inneren Abschnitt ziemlich gut verknöchert und $28 \mathrm{~mm}(0,84 \mathrm{~mm})$ lang, während die Ohrkapsel bereits in weiterer Ausdehnung verknöchert ist. Das $15 \mathrm{~mm}(0,45 \mathrm{~mm})$ lange Septum posterius und $23 \mathrm{~mm}(0,69 \mathrm{~mm})$ lange Septum laterale sind dagegen hier noch nicht völlig ossifiziert.

\section{Foramen ovale.}

Man hat angenommen : Das Foramen ovale ist längere Zeit an der schon verknorpelten Ohrkapsel sekundär zu Stande (Parker ('70), Wiedersheim ('77) u. a.) gekommen. Stöhr ('86) aber kam zuerst zur Ansicht, dass das Foramen von Anfang an als eine nicht verknorpelnde Spalte auftritt. Winslow ('98), Fuchs ('07), Okajima ('12), Stadtmüller ('25) u.a.m. schlossen sich auch dieser Ansicht an. Mein Befund stimmt auch vollständig mit diesen überein. Gaupp ('93) sagt von Rana fusca: „Durch den freien lateralen Rand der Basalplatte, den medial-ventralen Rand der Schale am äusseren Bogengange und die beiden basikapsulären Verbindungen wird eine grosse am Boden der Ohrkapsel gelegene Lücke begrenzt, die ich als ,, primäres Foramen ovale“ bezeichne. „, Beim Hyla arborea tritt das Foramen ovale zuerst 
im Stadium 1. (10 $\mathrm{mm}$ Gesamtlänge) auf, wobei dic Ohrkapsel noch nicht vollständig ausgebildet und gar nicht verknorpelt ist. Das Foramen liegt als eine lange grosse Lücke am lateralen ventralen Umfang der Kapsel und ist durch das lockere Bindegewebe geschlossen ; seine Längsachse ist von vorn nach hinten gerichtet. Beim Stadium $2(17 \mathrm{~mm}$ Gesamtlänge) ist es oval geformt und liegt horizontal, gegen vorher, ventral vom äusseren Bogengang; seine Ebene steht ungefähr senkrecht. Beim Stadium 3 (31 mm Gesamtlänge) ist es viel länger geworden und ist durch das Operkulargewebe geschlossen; seine Ebene ist ziemlich stark von vorn oben nach hinten unten geneigt. In diesem Stadium ist die Ohrkapsel ziemlich gut gebildet, wenngleich die Medialwand noch nicht vollständig geschlossen ist. Beim Stadium 4 (35 mm Gesamtlänge) ist es in der Länge kürzer geworden und liegt am kaudoventralen Umfang der lateralen Ohrkapselwand. Er enthält jetzt die erste kleine Operkularplatte in seinem linteren. Umfang; dabei kommt ein kurzer Stab der Columella vor, der mit dem unteren vorderen Rande des Foramen vorn vom Operculum in Verbindung steht. Im Stadium 5 (45 mm Gesamtlänge) zeigt das Foramen sich als ein fast ovales Fenster, liegt fast vertikal am lateralen ventralen Umfang der lateralen Kapselwand und ist durch den schalleitenden Apparat ziemlich genau geschlossen. Das Operculum ist schon gut verknorpelt, während die Columella noch nicht knorpelig und durch den sehr kurzen schmalen Stiel, den Stilus columellae, mit dem ventralen vorderen Rande des Foramen verbunden ist. Dieses Foramen ist oval und sein ventraler oraler Randteil ragt schon ziemlich deutlich in das Lumen hervor; seine Längsachse ist etwas von vorn lateral nach hinten medial gerichtet. Beim Stadium 6 (15 mm Stammlänge, gleich nach der Metamorphose) ist das Foramen länglich vom kaudomedialen Ende orolateralwärts ausgedehnt, so dass die Längsachse etwas von vorn lateral nach hinten medial gerichtet ist; sein ventraler oraler Rand ragt als eine abgerundete längliche Platte, Stilus columellae, in die Lücke hinein. Bei keinem Stadium konnte ich bisher eine im lateroventralen Abschnitt völlig kmorpelig geschlossene Lücke feststellen. Beim Stadium 7 (30 mm Stammlänge, ausgewachsenes Tier) ist das Foramen viel grösser geworden als beim vorbergehenden Stadium, liegt am lateroventrokaudaleì Abschnitt der Ohrkapselwand und wird jetzt ziemlich genau vom schon. vollständig ausgebildeten schalleitenden Apparat ausgefüllt; sein ventraler oraler Rand steigt sehr deutlich nach lateroorodosal auf uid geht in den hinteren Rand der Columella über. So steht die Ebene dieses Fensters yon vorm oben medial nach hinten unten lateral geneigt, die Längsachse dagegen von 
vorn oben lateral nach hinten unten medial gerichtet. Das Operculum verwächst in seinem medialen Rande mit dem hinteren Rande des Foramen und ragt in seiner hinteren Wand vom hinteren Rand des Foramen nach kaudal hinaus. Die Columellarplatte, Pars interna columellae, ragt, wie schon gesagt, direkt vom ventralen vorderen Rand des Foramen nach medial hervor und breitet sich auch horizontal von vorm nach hinten aus. Sein vorderes Ende erstreckt sich direkt zum vorderen Rand des Foramen und verwächst innig mit diesem.

\section{Schalleitender Apparat.}

Über die Entwicklung des schalleitenden Apparates bei den Wirbeltieren sind verschiedene Ansichten geäussert worden.

Rathke ('32) betrachtete das Operculum sowie die Columella des Frosches als die Bildung der Labyrinthwand; in der späterer Arbeit bei Natter ('39) hat er aber die Columella rom zweiten Veszeralbogen ableiten lassen. Nach Reichert ('38) entstammt bei den Amphibien das Operculum aus dem knorpeligen Labyrinth, die Columella dagegen vom oberen Teil des Zungenbeinbogens. Beim Frosch (Common Frog) sagt Parker ('70), dass das Operculum ein "periotic segment" ist, die Columella dagegen aus dem zweiten Viszeralbogen abstammt. Er ('76) sagt noch: "I yet hold, most tenaciously, to my old view as to the homologies of the parts ; namely, that the "Stapes" is a periotic, and the whole complex "Columella" a hyoidean element." Für Anuren haben Villy ('90) und Killian ('90) bestätigt, dass das Operculum eine rein labyrinthäre Bildung sei und die Columella aber aus einem Strang entsteht, der anfangs von der Labyrinthkapsel zum Quadratum zieht und von der Ohrkapsel aus verknorpelt. Gaupp ('93) sagt auch bei Rana fusca: „,Alle neueren Ansichten über die Entwicklung des betreffenden Apparates stimmen überein, und zwar das Operculum und die Pars interna plectori entstehen in der sekundären Fenestra ovalis voneinander vollständig und das Operculum und die Columella sind von der Ohrkapsel herzuleiten." Die obigen verschiedenen Ansichten lassen sich etwa folgendermassen zusammenfassen : Ersteus hat der schalleitende Apparat einen rein labyrinthären Ursprung und zweitens einen gemischten, labyrinthären und hyalen.'

Bei der: Ausbildung der vorkriorpeligen Ohrkapsel meines Tieres bleibt an seinem lateroventralen : Unfáng eine weite längliche, bald rrachher aber sehr verengende Lücke, die später durch den schalleitenden Apparat verschlossen wird. Das Operkulargewebe kommt zuerst im Stadium 3 '(31 mm Gesamtlänge) vor. Beim Stadium 4 (35 mm Gesamt- 
länge) findet man schon das vorknorpelige Operculum, das ungefähr vertikal, von vorn nach hinten gerichtet steht; die Columella wächst dabei als ein kleiner Fortsatz von seinem oberen vorderen Rand hervor und liegt im vorderen Umfang des Foramen ovale. Beim Stadium 5 (45 mm Gesamtlänge) ist das Operculum plötzlich stark entwickelt und schon ziemlich gut verknorpelt. Diese unregelmässig geformte Operkularplatte steht mit seiner vorderen ventralen Spitze etwas nach medial gerichtet und liegt ungefähr sagittal im hinteren Abschnitt des Foramen. Im Stadium 6 (15 mm Stammlänge, gleich nach der Metamorphose) zeigt das Operculum keine wesentliche Veränderung; es liegt ungefähr vertikal, aber mit seiner Spitze nach vorn unten medial gerichtet. Beim Stadium 7 (30 mm . Stammlänge, ausgewachsenes Tier) ist das Operculum ungefähr vollständig ausgebildet und stellt ein länggestrecktes, halbkugliges Gebilde dar, das von vorn gesehen fast oval erscheint und mit seiner Spitze nach vorn aussen gerichtet steht. Sein medialer Rand ist mit dem hinterem Rand des Foramen fast knorpelig verwachsen und ragt balbkuglig uach kaudal vom hinteren Rand des letzteren hinaus; dieses Verhältnis war beim vorigen Stadium noch nicht festzustellen. Der untere abgerundete Rand des Operculum erreicht dagegen den Unterrand des Foramen nicht, vielmehr bleibt dazwischen eine schmale Spalte übrig. Gaupp ('93) sagt bei Rana fasca : ,, Das Operculum ist eine selbständige Verknorpelung des Gewebes, das den grösseren hinteren Abschnitt des sekundären Foramen ovale verschliesst. Dieselbe findet schon ziemlich früh statt (Larven von ca. $30 \mathrm{~mm}$ )." Neuerdings fand ich auch bei Megalobatrachus japonicus ein gleiches Verhalten des Operculums. Ebenso auch zeigen die jüngeren Exemplare von Hyla immer eine selbständige Verknorpelung des Operkulargewebes, das das Foramen ovale verschliesst, während beim erwachseuen Tier (Stadium 7) das Operculum eine deutliche Veränderung zeigt und mediokaudalwärts mit der Kapselwand zusammenhängt. So hat das Operculum sich allmählich mehr kaudalwärts ausgewachsen und ist nach medial mit dem hinteren Rande des Foramen verwachsen. Dieser Zustand erklärt, dass im ausgewachsenen Tier das Operculum nach seinem relativ kurzen Verlaufe mit dem Rande des Foramen verwachsen ist.

Nie ist es mir gelungen, wie schon gesagt, irgend einen knorpeligen Zusammenhang des Operculums mit der Labyrinthkapsel und auch in ganzen Entwicklungsverlaufe eime Gliederung der Anlage des Operculums, was auf dessen gemischte Herkunft hinweist, zu finden. Daher kann man sagen, dass das Operculum durch die selbständige Verknorpelung des Operkulargewebes entsteht und genetisch aus der Obrkapselwand herzuleiten ist. 
Bezüglich der Anlage der Columella sagt Gaupp ('93) bei Rani fusca: ,Ebensowenig reicht aber das Operculum bis an den eigentlichen Vorderrand des Foramen ovale. Hier hat sich vielmehr ein zweites Gebilde, die Columella, angelegt. Dieselbe stellt zunächst noch eimen kurzen Stab vor, der von hinten innen nach vorn aussen gerichtet, dicht unter dem äusseren Bogengang liegt." ,, Die Klarheit der Verhältnisse erleidet nur dadurch eine Störung, dass der untere Umfang des Stabes mit dem unteren Rande des Foramen ovale vor dem Operculum in Verbindung steht. So schliesst das Foramen scheinbar schon dicht von dem Vorderrand des Operculum ab, und erst die genauere Betrachtung zeigt, dass hier nur eine schmale Knorpelzunge (Crista praeopercularis) vor dem Operculum die Columella mit dem unteren Umfange des Foramen verbindet." Bei meinem Tier findet man schon im Stadium 4 (35 mm Gesamtlänge) einen vorknorpeligen Fortsatz in vorderen Abschnitt des Foramen ovale. Dieser kleine Fortsatz ragt von dem unteren vorderen Rande des Foramen hervor und tritt mit seinem kleinen Stiel vorn vom Operculum in die Lücke ein. Das Operculum ist dabei gut gebildet. Im Stadium 5 (45 $\mathrm{mm}$ Gesamtlänge) befindet sich die Columella als ein vorknorpeliges dreieckiges Plättchen am vorderen Abschnitt des Foramen ; sie wächst mit seinem kleinsten Stiel von dem unteren vorderen Rand des Foramen heraus. Dieses Plättchen ist ungefähr horizontal von vorn nach hinten gerichtet und legt sich mit seinem hinteren spitzen Ende dem vorderen oberen Rand des Operculums an. Beim Stadium 6 (15 mm Stammlänge, gleich nach der Metamorphose) hat sich die Columella beträchtlich umgeformt, ist aber noch nicht ganz verknorpelt und stellt eine ziemlich grosse, horizontal liegende Platte dar, die mit ihrem stark nach innen abgerundeten, breiten Stiel vom unteren vorderen Rand des Foramen von lateral in die Lücke hineinragt und sich nur nach hinten bis zum mittleren Abschnitt des vorderen Randes der Operkularplatte erstreckt. So wird das Foramen ovale scheinbar in seinem unteren vorderen Umfang ganz von dem breiten Stilus columellae geschlossen. Beim Stadium 7 (30 mm Stammlänge, ausgewachsenes Tier) ist die Columella beträchtlicher gewachsen und erscheint hier zum erstenmal als eine fast vollkommen ausgebildete Kolumellarplatte, die jetzt völlig verknorpelt und sehr lang, mehrfach geknickt ist und sich von der Ohrkapsel nach aussen bis zum Trommelfell erstreckt. Die schon vollständig gebildete Pars interna columellae krümmt sich vom unteren vorderen Rand des Foramen ovale direkt nach innen und steht fast horizontal von vorn nach hinten gerichtet. Sie hat jetzt den konkaven, oberen inneren Umfang des Operculums und zugleich den vorderen Rand 
des Foramen erreicht. Die Pars media setzt sich mit seinem kleinen Stiel in den schon verknöcherten vorderen äusseren Rand der Pars interna columellae fort, zieht erst nach aussen oben und geht damn in rechtwinkliger Kuickung nach unten in die Pars externa über. Die Pars exterma stellt einen sehr langen Stab dar, ist lateroventrooralwärts gerichtet und setzt sich mit seinem spitzigen Ende in das Trommelfell fort. So schliesse ich mich bezüglich der Entstehung der Columella auch der Feststellung vou Gaupp an, indem ich irgendwelche Verhältnisse nicht gefunden habe, die auf eine Beziehung des Hyoidbogens und des Operculums bei der Knorpelbildung der Columella in dem Foramen hinweisen. Daher möchte ich behaupten, dass die Columella bei Hyla arborea aus der Ohrkapsel stammt und das Operculum durch die. selbständige Verknorpelung des Operkulargewebes entsteht und auch genetisch aus der Ohrkapsel abstammt.

\section{Ergebnisse.}

Die Hauptresultate der vorliegenden Arbeit lassen sich folgendermassen zusammenfassen :

1. Die Ohrkapsel stamimt von dem verdichteten periotischen Gewebe ab, das sich zum periotischen Knorpel differenziert. Zum Aufbau der Ohrkapsel wird jedoch nicht nur dieses Gewebe verwendet, sondern es beteiligt sich auch daran noch ein Teil der Basalplatte.

2. Bei den jungen Larven steht die Basalplatte durch die basikapsulare Verbindung mit der Anlage der Ohrkapsel in Zusammenhang. Die Verknorpelung der Ohrkapsel beginnt einmal in einer parachordal gelegenen Basalplatte und dann in einem oberen lateralen Teil des lateralen Bogenganges, so dass zwischen beiden ein Teil der Kapsel unterbrochen ist, der bei fortschreitender Verknorpelung zu einer Lücke, dem Foramen ovale, sich verengt.

3. Die mediale Kapselwand wird zuletzt gebildet. Die drei Foramina acustica und die zwei Foramina perilymphatica sind hier zu fiuden, von denen das Foramen acusticum posterius noch ein kleines Foramen acusticum medium zum Durchtritt des Ramus medius von sich abscheidet, der immer für die Lagena bestimmt ist.

4. Die Knorpelbildung der Ohrkapsel beginnt viel später als bei den Urodelen. So tritt die Bildung des echten Knorpels zuerst gleich vor der Metamorphose auf und schreitet gleichzeitig nach den versohiedenen Stellen der Kapsel fort ; die Stellen, wa die stärkste Knorpelbildung sich zeigt, sind der obere laterale Teil des lateralen Bogenganges 
Die Ohrkapsel und der schalleitende Apparat von Hyla arborea japonica.

und der ventrale Wandteil in der Nähe der Basalplatte, während die Medialwand sehr schwach verknorpelt ist.

5. Das Foramen acusticum posterius wird durch einen horizontalen Knorpelbalken in zwei Löcher zerlegt, von denen das obere das eigentliche Foramen acusticum posterius, das untere das Foramen medium darstellt.

6. Das Operculum entsteht aus der selbständigen Verknorpelung des Operkulargewebes, das von vornherein zur Labyrinthkapsel gehört. Die Columella wächst anfangs vom unteren vorderen Rand des Foramen ovale und steht im späteren Stadium knorpelig mit dem Hinterrande des Foramen in Verbindung. Die Columella verknorpelt sich selbständig ziemlich später als das Operculum selbst.

7. Das Foramen ovale tritt im sehr früheren Stadium als eine grosse Lücke an dem lateroventralen Umfang der lateralen Kapselwand auf und wird durch die Bindegewebesschicht geschlossen. Die Ebene des Foramen steht anfangs horizontal von vorn nach hinten gerichtet, ist im späteren Stadium sehr vergrössert und neigt sich von vorn oben lateral nach hinten unten medial. Der schalleitende Apparat erscheint zuerst in seinem vorderen und hinteren Umfang. Zuletzt wird das Foramen ein sehr grosses ovales Fenster an dem lateroventrokaudalen Umfang der lateraleu Kapelwand und ist durch den schon verknorpelten schalleitenden Apparat geschlossen, mit seiner Längsachse von vorn oben lateral nach hinten unten medial gerichtet; zwischen dem Apparat und der Fensterwand bleibt eine sehr verengte ringförmige Lücke übrig, das sekundäre Foramen ovale, das mit seiner Längsachse von vorn oben lateral nach hinten unten'medial geneigt steht.

Zum Schluss möchte ich Herrn Prof. K. Okajima für seine dauernde Unterstützung bei dieser Arbeit ineinen herzlichsten Dank aussprechen.

\section{Literatur.}

1. Cords, E., Die Entwicklung der Paukenhöhle yon Iacerta agilis. Ein Beitrag zur Lehre vom schalleitenden Apparat der Wirbeltiere. Anat. Hefte. Bd. 38. 1909.

2. Ecker-Gaupp, Anatomie des Frosches. Braunschweig 1904.

3. Fuchs, H., Ứber die Fntwicklung des Operculum der Urodelen und des Distelidiums ("Columella" auris) einiger Reptilien; Anat. Anz. Ergänz. f. Bd. 30: 1907. 
4. Gegenbaur, C., Vergleichende Anatomie der Wirbeltiere. Jena 1909.

5. — Die Metamerie des Kopfes und die Wirbeltheorie des Kopfskelettes. Morph. Jahrb. Bd. 13.1888.

6. Gaupp, E., Das Chondrocranium von Iacerta agilis. Ein Beitrag zum Verständnis des Amniotenschädels. Anat. Hefte. Bd. 15. 1900.

7. —, Die Fntwicklung des Kopfes. Hertwig's Handbuch d. vergl. u. exper. Entw. 1906.

8. - Ontogenese und Phylogenese des schalleitenden Apparates bei den Wirbeltieren. Ergeb. d. Anat. u. Entw. Bd. 8. 1898-99.

9. —- Primordial-Cranium und Kieferbogen von Rana fusca. Morph. Arb. Bd. 2. 1893.

10. Hertwig, O., Handbuch d. vergl. u. exper. Entwicklungslehre. Bd. 2. II. Teil. 1906.

11. Hafferl, A., Über das knorpelige Neurocranium des Gecko. Ein Beitrag zur Entwicklungsgeschichte des Reptilienschädels. Zeitschr. f. Anat. u. Entw. Bd. 62. 1921.

12. Hasse, C., Das knöcherne Iabyrinth der Frösche. Anat. Stud. 1. Ieipzig 1873 (Ref. bei Gaupp).

13. Koth, K., Entwicklungsgeschichtliche Ontersuchungen über das Zungenbein und die Ohrknöchelchen der Anuren. Arch. f. Naturgeschichte. Jahrg. 76. 1906 (Ref. bei Klaauw).

14. Klaauw, C. J., Bau und Entwicklung der Gehörknöchelchen. Ergeb. der Anat. und Entw. Bd. xxv. 1924.

15. Migawaki, S., Das Vorhandensein des Foramen acusticum medium an der Ohrkapsel von Anuren. Folia Anat. Japon. Bd. 5. 1927.

16. - U̇ber die Foramina acustica media an der Ohrkapsel von Reptilien. Folia Anat. Japon. Bd. 6. 1928.

17. Nick, J., Das Kopfskelett von Dermochelys coriacea I. Zool. Jahrb. Abt. Anat. Bd. 33. 1912.

18. Okajima, K., Contribution a l'étude de l'organe de l'ouie chez les Urodèles. Arch. de Biol. Tom. 23. 1910.

19. — Die Entwicklung des Gehörorgans von Hynobius. Anat. Hefte. Bd. 45. 1912.

20. - - Über das Vorkommen von zwei Foramina perilymphatica bein Urodelenohr. Anat. Anz. Bd. 38. 1911.

21. Ogushi, K., Anatomische Studien an der japanischen dreikralligen Lippenschildkröte (Trionyx japonicus). 1. Mitteilung. Morph. Jahrb. Bd. 43. 1911.

22. Parker, W. K., On the Structure and Development of the Skull of the Common Frog. Philos. Transact. Vol. 161. 1870. (Ref. bei. G a u p p).

23. Peter, K., Die Entwicklung und funktionelle Gestaltung des Schädels von Ichthyophis glutinosus. Morph. Jahrb. Bd. 25. 1898.

24. Retzius, G., Das Gehörorgan der Wirbeltiere. 1. Das Gehörorgan der Fische und Amphibien. Stockholm 1881.

25. — Zur Kenntnis des inneren Gehörorgans der Wirbeltiere. Arch. f. Anat. u. Phys. Anat. Abt. 1880.

26. Reed, H. D., The morphology of the sound-transmitting apparatus in caudate amphibia and its phylogenetic significance. Journ. Morph. 33. 1920.

27. Stadtmüller, F. Zur Entwicklungsgeschichte des Kopfskelettes der Salamandra maculosa. Zeitschr. f. Anat. u. Entw. Bd. 75. 1925. 
Die Ohrkapsel und der schalleitende Apparat von Hyla arborea japonica.

28. Stöhr, P., Zur Entwicklungsgeschichte des Anurenschädels. Zeitschr. f. wiss. Zool. Bd. 36. 1881 .

29. Villy, F., The development of the ear and the accesory organs in the Common Frog. Quar. Journ. of Micr. Science. Vol. 30. 1890.

30. Versluys, J., Die mittlere u. äussere Ohrsphäre der Lacertilia u. Rhychocephalia. Zool. Jahrb. Abt. f. Anat. Bd. 12. 1898.

31. — Zur Entwicklung der Columella auris bei den Iacertilien. Ein Beitrag zur Kenntnis des schalleitenden Apparates und des Zungenbeinbogens bei den Sauropsiden. Zool. Jahrb. Abt. f. Anat. Bd. 19. 1903.

32. Wiedersheim, R., Das Kopfskelett der Urodelen. Morph. Jahrb. Bd. 3. 1877.

33. —, Vergleichende Anatomie der Wirbeltiere. Ieipzig 1898.

34. Winslow. G. M., The chondrocranium in the Ichthyopsida. Tufts college studies. No. 5. 1898. (Ref. bei Okajima). 